\title{
Free energy estimation of short DNA duplex hybridizations
}

Dan Tulpan ${ }^{1 *}$, Mirela Andronescu², Serge Leger $^{1}$

\begin{abstract}
Background: Estimation of DNA duplex hybridization free energy is widely used for predicting cross-hybridizations in DNA computing and microarray experiments. A number of software programs based on different methods and parametrizations are available for the theoretical estimation of duplex free energies. However, significant differences in free energy values are sometimes observed among estimations obtained with various methods, thus being difficult to decide what value is the accurate one.

Results: We present in this study a quantitative comparison of the similarities and differences among four published DNA/DNA duplex free energy calculation methods and an extended Nearest-Neighbour Model for perfect matches based on triplet interactions. The comparison was performed on a benchmark data set with 695 pairs of short oligos that we collected and manually curated from 29 publications. Sequence lengths range from 4 to 30 nucleotides and span a large GC-content percentage range. For perfect matches, we propose an extension of the Nearest-Neighbour Model that matches or exceeds the performance of the existing ones, both in terms of correlations and root mean squared errors. The proposed model was trained on experimental data with temperature, sodium and sequence concentration characteristics that span a wide range of values, thus conferring the model a higher power of generalization when used for free energy estimations of DNA duplexes under nonstandard experimental conditions.

Conclusions: Based on our preliminary results, we conclude that no statistically significant differences exist among free energy approximations obtained with 4 publicly available and widely used programs, when benchmarked against a collection of 695 pairs of short oligos collected and curated by the authors of this work based on 29 publications. The extended Nearest-Neighbour Model based on triplet interactions presented in this work is capable of performing accurate estimations of free energies for perfect match duplexes under both standard and non-standard experimental conditions and may serve as a baseline for further developments in this area of research.
\end{abstract}

\section{Background}

Predicting the stability of a DNA duplex from base sequences is a well studied problem nowadays. Nevertheless, the accuracy of DNA duplex stability predictions largely varies with sequence length, base composition and experimental conditions. The Thermodynamic Nearest-Neighbour (TNN) Model [1] is a state-of-theart approach that is used to estimate the stability of a single or a pair of DNA (or RNA) molecules based on pairwise base interactions and structural conformations. A large collection of thermodynamic nearest-neighbour

\footnotetext{
* Correspondence: dan.tulpan@nrc-cnrc.gc.ca

${ }^{1}$ National Research Council of Canada, Institute of Information Technology,

100 des Aboiteaux Street, Suite 1100, Moncton, NB, E1A 7R1, Canada
}

parameters were acquired by interpolation of results obtained from various experimental processes like NMR [2] and optical melting studies [1,3]. The accuracy of computing free energies for DNA duplexes is an important aspect for all prediction methods, considering their direct application for selecting, for example, microarray probes that perfectly hybridize with their complements within a pre-specified hybridization interval, while avoiding self-hybridization for each probe [4]. Here we select four widely used, publicly available computer programs that implement the TNN Model using large numbers of experimentally derived thermodynamic parameters, namely: the MultiRNAFold v2.0 package $[5,6]$ with two sets of thermodynamic parameters, the

C Biomed Central

(c) 2010 Tulpan et al; licensee BioMed Central Ltd. This is an Open Access article distributed under the terms of the Creative Commons Attribution License (http://creativecommons.org/licenses/by/2.0), which permits unrestricted use, distribution, and reproduction in any medium, provided the original work is properly cited. 
Vienna Package v1.8.1 [7] and the UNAFold v3.5 package [8].

The MultiRNAFold package (including the PairFold program for duplexes) predicts the minimum free energy, suboptimal secondary structures and free energy changes of one, two, or several interacting nucleic acid sequences. The thermodynamic model for the thermodynamic stability of a joint secondary structure for two DNA or RNA molecules at a given temperature is performed similarly to that of a single molecule [9], except that an intermolecular initiation penalty is added. The PairFold algorithm uses dynamic programming to calculate minimum free energy secondary structures and runs in time cubic in the lengths of the input sequences $\left(\Theta\left(n^{3}\right)\right)$. PairFold uses RNA thermodynamic parameters from the Turner Laboratory [10] and DNA thermodynamic parameters from the Mathews and SantaLucia laboratories [11,12].

The Vienna Package consists of a suite of computer programs and libraries for prediction of RNA and DNA secondary structures. Nucleic acid secondary structure prediction is done via free energy minimization using three dynamic programming algorithms for structure prediction: the minimum free energy algorithm of [13], which produces a single optimal structure, the partition function algorithm of [14], which calculates base pair probabilities in a thermodynamic ensemble, and the suboptimal folding algorithm of [15], which generates all suboptimal structures within a given energy range of the optimal energy.

UNAFold, the acronym for "Unified Nucleic Acid Folding", is a software package for RNA and DNA folding and hybridization prediction. UNAFold folds singlestranded RNA or DNA, or two single DNA or RNA strands, by computing partition functions for various states of hybridization. The partition functions will then help to derive base pair probabilities and stochastic samples of foldings or hybridizations. The package provides various energy minimization methods, which compute minimum free energy hybridizations and suboptimal foldings.

All three packages use similar dynamic programming algorithms for prediction of minimum free energy (MFE) and suboptimal structures and for partition function calculations. For the purposes of our work (i.e., DNA duplex MFE secondary structure prediction and free energy of hybridization), the main differences lie in the thermodynamic parameters used (SantaLucia or Mathews), and in the features considered (for example, the Vienna Package does not consider special types of poly-C hairpin loops in their model, whereas the other two packages do). Thus our first goal is to quantify the impact of these differences on the accuracy of DNA duplex free energy approximations. Throughout the paper, we use a set of measures that reflect the degree of similarity of calculated and experimental secondary structures and free energies. Based on these measures we quantify the accuracy of the predictions of the aforementioned programs using a collection of 695 experimental DNA duplex data that we collected from 29 publications.

We also introduce in this work an extended NearestNeighbour Model for perfect matches based on triplet interactions, that can approximate free energies for DNA duplexes under a wide range of temperatures, sodium and sequence concentrations. The model is similar to the one introduced in 1999 by Owczarzy et al. [16], the main difference residing in the inclusion of only triplet interactions for our model, rather than a mixture of singlets, doublets and triplets for the other. Thus, our second goal is to show that such a model outperforms simpler models based on doublet interactions and produces more accurate free energy approximations for DNA duplex hybridizations occurring in non-standard experimental environments (for example for different sodium concentrations or at different temperatures).

\section{Results and Discussion}

In this work, we compare similarities and correlations of free energy values calculated using three publicly available packages, namely MultiRNAFold, UNAFold and Vienna Package and a Nearest-Neighbour (NN) Model for perfect matches based on triplet interactions. For this purpose, we collected and used a data set with 695 pairs of short DNA sequences and we investigated what method produces the closest value to the experimental free energy and under what circumstances. We acknowledge the fact that not all sequence lengths are equally represented in the benchmark data set simply due to their availability and thus our analysis may apply better to shorter sequences. The majority $(91.37 \%)$ of experimental free energy calculations were obtained for perfect ( 0 mismatches) and almost perfect matches (1 mismatch), thus the current DNA parameter sets tend to have higher accuracy for close-to perfect match DNA duplexes. Another bias in the analysis may come from the fact that some authors have already tried to reconcile the existing differences in free energy model parameters $[17,18]$ by optimizing sets of DNA parameters using the same sequences already present in the benchmark data set.

\section{Comparison of absolute differences between experimental and estimated free energies (MFE_AD)} We begin the presentation of our results by introducing a measure that provides insights into "worst" and "best" estimates for minimum free energies. Thus, the first 
comparison involves the absolute differences between experimental and estimated free energies (MFE_AD) among all the methods for model evaluation (column 3 in Table 1) and model prediction (column 3 in Table 2). In an ideal scenario, the estimated free energy would equal the experimentally inferred one, nevertheless in practice we would settle for a low absolute difference. In both scenarios, namely the evaluation of free energy estimates and the evaluation of secondary structure predictions, the largest maximal MFE_AD $(18.4 \mathrm{kcal} / \mathrm{mol}$ in both) were obtained for the PairFold-Mathews method, while the minimal MFE_AD (13 $\mathrm{kcal} / \mathrm{mol}$ for EVAL-FE and $11.88 \mathrm{kcal} / \mathrm{mol}$ for EVAL-SS) corresponds to the UNAFold method (see Methods for details). The average differences for the EVAL-FE methods range between $2.41 \mathrm{kcal} / \mathrm{mol}$ (UNAFold) and $3.16 \mathrm{kcal} / \mathrm{mol}$ (Vienna
Package), while for the prediction methods the interval is slightly shifted towards zero. We also observed a similar improvement trend for MFE_AD standard deviations of EVAL-SS methods versus EVAL-FE methods, a phenomenon that can be explained by the intrinsic regression-based construction of the underlying DNA parameters used by each method.

\section{Comparison of root mean squared errors (RMSE)}

We measure the root mean squared error between experimentally determined and predicted free energies. In an ideal scenario where predicted values equal experimental values, the RMSE would be zero, thus the lower the RMSE value is, the closer the predicted values are to the experimental ones. Here, all methods produce comparably low RMSEs, the lowest EVAL-FE RMSE (3.876)

Table 1 Summary of features for the data sets used in this study

\begin{tabular}{|c|c|c|c|c|c|}
\hline Set & Num. duplexes & Seq. len. & $T[\mathrm{C}]$ & {$[\mathrm{Na}]^{+}[\mathrm{M}]$} & Seq. conc. [M] \\
\hline Aboul-ela et al. [32] & 34 & 16 & 25,50 & 1 & {$[11 e-6,440 e-6]$} \\
\hline Allawi et al.-1 [37] & 24 & $9-12$ & 37 & 1 & $1 \mathrm{e}-4$ \\
\hline Allawi et al.-2 [20] & 28 & $9-14$ & 37 & 1 & $1 e-4$ \\
\hline Allawi et al.-3 [21] & 22 & $9-14$ & 37 & 1 & $1 e-4$ \\
\hline Bommarito et al. [43] & 37 & $8-9$ & 37 & 1 & n.r. \\
\hline Breslauer et al. [26] & 12 & $6-16$ & 25 & 1 & n.r. \\
\hline Clark et al. [44] & 1 & 24 & 37 & 0.15 & $2.5 e-6$ \\
\hline Doktycz et al. [19] & 140 & 8 & 25 & 1 & 2e-6 \\
\hline Gelfand et al. [45] & 4 & 13 & 25 & 1 & $5 e-5$ \\
\hline LeBlanc et al. [46] & 7 & $10-11$ & 25 & 1 & $5 e-5$ \\
\hline Leonard et al. [22] & 5 & 12 & 25 & 1 & $4 e-4$ \\
\hline Lesnik et al. [39] & 14 & $8-21$ & 37 & 0.1 & $4 e-6$ \\
\hline Li et al. [23] & 12 & $8-10$ & 25 & 1 & $6.1 e-6$ \\
\hline Nakano et al. [40] & 21 & $6-14$ & 37 & 0.1 & $8 e-6$ \\
\hline Petruska et al.-1 [47] & 4 & 9 & 37 & n.r. & n.r. \\
\hline Petruska et al.-2 [36] & 2 & 30 & 37 & 0.17 & $1 e-4$ \\
\hline Peyret et al. [48] & 52 & $9-12$ & 37 & 1 & $1 e-4$ \\
\hline Pirrung et al. [49] & 2 & 25 & 25 & 0.1 & $1 e-6$ \\
\hline Plum et al. [50] & 2 & 13 & 25 & 1 & $6 e-6$ \\
\hline Ratmeyer et al. [51] & 2 & 12 & 37 & 1 & $6 e-6$ \\
\hline SantaLucia et al.-1 [29] & 23 & $4-16$ & 37 & 1 & $4 e-4$ \\
\hline SantaLucia et al.-2 [29] & 10 & 12 & 24.85 & 1 & $5 e-6$ \\
\hline Sugimoto et al.-1 [30] & 50 & $5-14$ & 37 & 1 & $5 e-6$ \\
\hline Sugimoto et al.-2 [38] & 1 & 8 & 37 & n.r. & $1 e-4$ \\
\hline Sugimoto et al.-3 [52] & 8 & $6-8$ & 37 & 1 & n.r. \\
\hline Tanaka et al. [34] & 126 & $12-25$ & 37 & 1 & $5 e-5$ \\
\hline Tibanyenda et al. [33] & 3 & 16 & 24.85 & 1 & $17.5 e-6$ \\
\hline Wilson et al. [35] & 3 & 11 & 25 & 0.4 & n.r. \\
\hline Wu et al. [53] & 48 & $5-11$ & 25,37 & 1 & $1 e-4$ \\
\hline TOTAL: & 695 & & & & \\
\hline
\end{tabular}

Each data set has the following characteristics: the number of sequence pairs (Num. duplexes), the length of the sequences (Seq. len), the experimental temperature measured in degrees Celsius for estimating free energies $(\mathrm{T})$, the sodium concentration measured in molar units ([Na $\left.]^{+}\right)$and the sequence concentration (Seq. conc). The set of 695 DNA duplexes contains: (i) 143 perfect match free energies measured at a temperature of $37^{\circ} \mathrm{C}$ and a sodium concentration of $1 \mathrm{M}$, (ii) 197 perfect match duplexes measured at a temperature of $25^{\circ} \mathrm{C}$ and a sodium concentration of $1 \mathrm{M}$, (iii) 7 perfect match duplexes measured at a temperature of $50^{\circ} \mathrm{C}$ and a sodium concentration of $1 \mathrm{M}$, and (iv) 348 duplexes with mismatches measured at various temperatures and sodium concentrations. Note: n.r. denotes values that have not been reported in the original documents. 
Table 2 Summary of results for free energy measurements obtained with EVAL-SS methods

\begin{tabular}{|c|c|c|c|c|c|c|c|c|}
\hline Method & Stats & MFE_AD [kcal/mol] & Pearson coeff. $(\mathbf{r})$ & & SSSI & Sens. & PPV & F-measure \\
\hline MultiRNAFold & $\min$ & 0.000 & 0.7565 & 4.35 & 40.00 & 0.1667 & 1 & 0.2857 \\
\hline \multirow[t]{6}{*}{ (Mathews) } & $q 1$ & 0.340 & & & 100.00 & 1.0000 & 1 & 1.0000 \\
\hline & median & 0.860 & & & 100.00 & 1.0000 & 1 & 1.0000 \\
\hline & mean & 2.681 & & & 95.83 & 0.9547 & 1 & 0.9711 \\
\hline & q3 & 3.590 & & & 100.00 & 1.0000 & 1 & 1.0000 \\
\hline & $\max$ & 18.400 & & & 100.00 & 1.0000 & 1 & 1.0000 \\
\hline & stddev & 3.429 & & & 10.56 & 0.1224 & 0 & 0.09236 \\
\hline MultiRNAFold & $\min$ & 0.000 & 0.7663 & 4.131 & 40.00 & 0.1667 & 1 & 0.2857 \\
\hline \multirow[t]{9}{*}{ (SantaLucia) } & q1 & 0.330 & & & 100.00 & 1.0000 & 1 & 1.0000 \\
\hline & median & 0.720 & & & 100.00 & 1.0000 & 1 & 1.0000 \\
\hline & mean & 2.528 & & & 96.44 & 0.9608 & 1 & 0.9747 \\
\hline & q3 & 3.510 & & & 100.00 & 1.0000 & 1 & 1.0000 \\
\hline & $\max$ & 17.200 & & & 100.00 & 1.0000 & 1 & 1.0000 \\
\hline & stddev & 3.269 & & & 10.23 & 0.1189 & 0 & 0.08966 \\
\hline & $\min$ & 0.000 & 0.7660 & 3.992 & 40.00 & 0.1667 & 1 & 0.2857 \\
\hline & q1 & 0.256 & & & 100.00 & 1.0000 & 1 & 1.0000 \\
\hline & median & 0.630 & & & 100.00 & 1.0000 & 1 & 1.0000 \\
\hline \multirow[t]{4}{*}{ UNAFold } & mean & 2.374 & & & 96.08 & 0.9571 & 1 & 0.9724 \\
\hline & q3 & 3.016 & & & 100.00 & 1.0000 & 1 & 1.0000 \\
\hline & $\max$ & 11.880 & & & 100.00 & 1.0000 & 1 & 1.0000 \\
\hline & stddev & 3.212 & & & 10.66 & 0.1231 & 0 & 0.09234 \\
\hline Vienna & $\min$ & 0.010 & 0.7630 & 3.667 & 5.882 & 0.0000 & 0.0000 & 0.0000 \\
\hline \multirow[t]{6}{*}{ Package } & q1 & 1.700 & & & 100.000 & 1.0000 & 1.0000 & 1.0000 \\
\hline & median & 2.330 & & & 100.000 & 1.0000 & 1.0000 & 1.0000 \\
\hline & mean & 3.025 & & & 95.210 & 0.9467 & 0.9856 & 0.9616 \\
\hline & q3 & 3.935 & & & 100.000 & 1.0000 & 1.0000 & 1.0000 \\
\hline & $\max$ & 15.400 & & & 100.000 & 1.0000 & 1.0000 & 1.0000 \\
\hline & stddev & 2.075 & & & 13.74 & 0.1581 & 0.1192 & 0.1387 \\
\hline
\end{tabular}

Summary of results for free energy measurements obtained with EVAL-SS methods. The p-values for the Pearson correlation test were less than 2.2e-16 in all cases.

and EVAL-SS RMSE (3.667) being obtained in both cases with Vienna Package (column 5 in Tables 3 and 4).

\section{Comparison of Pearson correlation coefficients $(r)$}

A correlation coefficient is traditionally defined as a symmetric, scale-invariant measure of association between two random variables, which takes values between -1 and 1 . The extreme values indicate a perfect positive (1) or negative (-1) correlation, while 0 means no correlation. Positive Pearson Product Moment correlations are observed for all methods when experimental and evaluated or predicted free energies are considered as random variables. The highest Pearson correlation coefficients $(\sim .75$ and $\sim .77)$ are consistently obtained with the PairFold-SantaLucia method for both EVAL-FE and EVAL-SS, closely followed by UNAfold, Vienna Package and PairFold-Mathews. A major and consistent deviation from the correlation line of approximately 8 $\mathrm{Kcal} / \mathrm{mol}$ for the data collected from Doktycz et al. [19] and a few other minor deviations for the data collected from four additional publications [20-23] were consistently noticed for all free energy calculation methods (see Figures 1 and 2). The majority of the deviations (e.g. Doktycz et al. [19]) may come from potentially different free energy interpolation functions used in those studies.

If we consider only perfect match data, the TNN-Triplets-PM Model (see Methods) is capable of estimating free energies that correlate better $(r=0.92)$ with experimental values (see Figure 3), than all the other methods, which show an average correlation coefficient $r=0.68$. We notice also an improvement in the RMSE for the TNN-Triplets-PM Model, compared to the other programs. To ensure that this improvement is due to the triplet aspect of the model rather than other confounding factors, we created a TNN-Doublets-PM Model that has been trained and evaluated on the same perfect match data set. A detailed description of the training and evaluation procedure is provided in Tables 5 and 6. For the complete data set with perfect matches measured at various temperatures and buffer concentrations, Figures 4, 5, 6, 7, 
Table 3 Summary of results for free energy measurements obtained with EVAL-FE methods

\begin{tabular}{|c|c|c|c|c|}
\hline Method & Statistics & $\begin{array}{c}\text { MFE_AD [kcal/ } \\
\text { mol] }\end{array}$ & $\begin{array}{c}\text { Pearson coeff. } \\
(\mathrm{r})\end{array}$ & RMSE \\
\hline MultiRNAFold & $\min$ & 0.0000 & 0.7352 & 4.418 \\
\hline \multirow[t]{6}{*}{ (Mathews) } & q1 & 0.300 & & \\
\hline & median & 0.800 & & \\
\hline & mean & 2.672 & & \\
\hline & q3 & 3.395 & & \\
\hline & $\max$ & 18.400 & & \\
\hline & stddev & 3.521 & & \\
\hline MultiRNAFold & $\min$ & 0.0000 & 0.7456 & 4.223 \\
\hline \multirow[t]{9}{*}{ (SantaLucia) } & $q 1$ & 0.330 & & \\
\hline & median & 0.680 & & \\
\hline & mean & 2.553 & & \\
\hline & q3 & 3.390 & & \\
\hline & $\max$ & 17.200 & & \\
\hline & stddev & 3.367 & & \\
\hline & $\min$ & 0.0000 & 0.7434 & 4.101 \\
\hline & q1 & 0.2528 & & \\
\hline & median & 0.6128 & & \\
\hline \multirow[t]{4}{*}{ UNAFold } & mean & 2.4110 & & \\
\hline & q3 & 2.9970 & & \\
\hline & $\max$ & 13.0000 & & \\
\hline & stddev & 3.319 & & \\
\hline Vienna & $\min$ & 0.0000 & 0.7413 & 3.876 \\
\hline \multirow[t]{6}{*}{ Package } & q1 & 1.820 & & \\
\hline & median & 2.440 & & \\
\hline & mean & 3.167 & & \\
\hline & q3 & 3.965 & & \\
\hline & $\max$ & 15.400 & & \\
\hline & stddev & 2.236 & & \\
\hline
\end{tabular}

Summary of results for free energy measurements obtained with EVAL-FE methods. The $p$-values for the Pearson correlation test were less than 2.2e-16 in all cases.

8 and 9 show that our TNN-Triplets-PM Model consistently produces better correlations and RMSEs, when we run a random design experiment using 10000 randomly selected subsets with $67 \%$ duplexes $(228$ perfect match duplexes) used for training and 33\% duplexes (112 perfect match duplexes) used for testing. The same high correlations can be observed when running the TNN-TripletsPM Model on perfect match duplex free energies measured at a temperature of $25^{\circ} \mathrm{C}$ and $1 \mathrm{M}$ sodium concentration, while for perfect match free energies measured at $37^{\circ} \mathrm{C}$ and $1 \mathrm{M}$ sodium concentration, the other models produce better but still comparable correlations (0.9) and RMSEs (0.7) with the TNN-Triplets-PM Model.

Comparison of secondary structure similarity indexes of experimental and predicted secondary structures (SSSI)

The accuracy of secondary structure prediction for various methods can be evaluated by using the newly introduced measure described in equation 5. The SSSI measure simply calculates the percentage of correctly predicted secondary structure bonds corresponding to the positions in each secondary structure (corresponding to each sequence in the duplex) that match the position in the experimental secondary structure, normalized by the sum of sequence lengths. Comparable mean SSSI values were produced by all methods with a maximal value of $96.44 \%$ attained by PairFold-SantaLucia. The lowest value (95.21\%) was obtained with Vienna Package (see column 6 in Table 2). All methods have large standard deviation for SSSI values, thus suggesting a wide sample distribution.

\section{Comparison of SENS, PPV and F for predicted secondary structures}

The analysis of the variation for sensitivities and F-measures with respect to sequence length and GC content percentages reveals a common pattern for all prediction methods. Mean sensitivities higher than 0.9 and mean Fmeasures higher than 0.95 were obtained for all methods and all sequence lengths with one exception. For sequences of length 10 a major drop in sensitivities and Fmeasures can be observed (see Figures 10 and 11). The main cause for the abrupt drop in sensitivities seem to apply mostly for sequences whose experimentally determined secondary structures contain two consecutive mismatches (collected from [23]), thus partially supporting the hypothesis that the prediction models under investigation seem to be optimized to produce better results for almost complementary pairs of DNA sequences. Next we look at how GC content \% impacts the accuracy of prediction for the methods under consideration. While sensitivities and F-measures are higher than 0.9 for all methods for a wide range of GC content \% intervals (e.g. $0 \%-10 \%$, $40 \%-100 \%$ ), there are values for which sensitivities and Fmeasures drop under 0.9 for sequences with GC content percentages in the range 10\% - 40\%. While Pairfold-Mathews, Pairfold-SantaLucia and UNAFold generate predictions with sensitivities higher than 0.9 for sequences with GC content percentages in the range $20 \%-30 \%$, the Vienna Package has a mean sensitivity of only 0.8 . For 3 out of 4 methods, the PPV equals 1 (maximum), while for the remaining one, namely the Vienna Package slightly lower mean values $(0.98)$ were obtained.

\section{Comparison of free energy parameters for DNA doublets measured at $37^{\circ} \mathrm{C}$ and $1 \mathrm{M}$ sodium concentration}

Table 2 presents the estimated free energy parameters for DNA doublets measured at $37^{\circ} \mathrm{C}$. The set of 10 parameters corresponds to the best set obtained with the procedure explained in Table 6 . We compared our set of $\mathrm{NN}$ free energy parameters at $37^{\circ} \mathrm{C}$ with eight other sets of parameters reported by SantaLucia [18], namely the sets obtained by Gotoh [24], Vologodskii 
Table 4 Estimated free energy parameters

\begin{tabular}{|c|c|c|c|c|c|c|c|c|c|}
\hline ID & Doublet & $\Delta G_{37}^{o}$ & {$[\mathrm{kcal} / \mathrm{mol}]$} & Counts & ID & Doublet & $\Delta G_{37}^{o}$ & {$[\mathrm{kcal} / \mathrm{mol}]$} & Counts \\
\hline 1. & $\mathrm{AA} / \mathrm{TT}$ & & -0.838948 & 84 & 6. & $\mathrm{CC} / \mathrm{GG}$ & & -1.698997 & 74 \\
\hline 2. & $\mathrm{AC} / \mathrm{TG}$ & & -1.394988 & 102 & 7. & $\mathrm{CG} / \mathrm{GC}$ & & -0.967002 & 106 \\
\hline 3. & $\mathrm{AG} / \mathrm{TC}$ & & -1.323547 & 102 & 8. & $\mathrm{GA} / \mathrm{CT}$ & & -0.938327 & 101 \\
\hline 4. & AT/TA & & -0.375235 & 130 & 9. & $\mathrm{GC} / \mathrm{CG}$ & & -0.711466 & 126 \\
\hline 5. & CA/GT & & -1.406794 & 95 & 10. & TA/AT & & -0.144092 & 136 \\
\hline ID & Triplet & $\Delta G_{37}^{o}$ & {$[\mathrm{kcal} / \mathrm{mol}]$} & Counts & ID & Triplet & $\Delta G_{37}^{o}$ & {$[\mathrm{kcal} / \mathrm{mol}]$} & Counts \\
\hline 1. & $\mathrm{AAA} / \mathrm{TT}$ & & -0.844597 & 10 & 17. & CAG/GTC & & -1.625284 & 23 \\
\hline 2. & $\mathrm{AAC/TTG}$ & & -1.841904 & 19 & 18. & CCA/GGT & & -1.568813 & 18 \\
\hline 3. & $\mathrm{AAG} / \mathrm{TTC}$ & & -1.201194 & 17 & 19. & $\mathrm{CCC} / \mathrm{GGG}$ & & -2.396507 & 17 \\
\hline 4. & AAT/TTA & & -0.991596 & 19 & 20. & $\mathrm{CCG} / \mathrm{GGC}$ & & -1.888906 & 22 \\
\hline 5. & ACA/TGT & & -1.121939 & 20 & 21. & $\mathrm{CGA} / \mathrm{GCT}$ & & -1.668273 & 19 \\
\hline 6. & ACC/TGG & & -1.793995 & 23 & 22. & CGC/GCG & & -2.195726 & 23 \\
\hline 7. & ACG/TGC & & -1.615048 & 30 & 23. & CTA/GAT & & -0.871636 & 40 \\
\hline 8. & $\mathrm{ACT} / \mathrm{TG} \mathrm{A}$ & & -0.781693 & 23 & 24. & $\mathrm{CTC} / \mathrm{GAG}$ & & -1.198450 & 16 \\
\hline 9. & $\mathrm{AGA} / \mathrm{TCT}$ & & -1.103536 & 15 & 25. & $\mathrm{GAA} / \mathrm{CTT}$ & & -1.317278 & 18 \\
\hline 10. & AGC/TCG & & -1.528461 & 36 & 26. & $\mathrm{GAC} / \mathrm{CTG}$ & & -1.498999 & 29 \\
\hline 11. & AGG/TCC & & -1.323278 & 18 & 27. & GCA/CGT & & -1.454430 & 21 \\
\hline 12. & ATA/TAT & & -0.562379 & 46 & 28. & GCC/CGG & & -1.973081 & 24 \\
\hline 13. & ATC/TAG & & -1.157521 & 29 & 29. & GGA/CCT & & -1.696158 & 20 \\
\hline 14. & ATG/TAC & & -1.263601 & 26 & 30. & GTA/CAT & & -1.158422 & 32 \\
\hline 15. & CAA/GTT & & -0.988509 & 16 & 31. & TAA/ATT & & -0.519499 & 27 \\
\hline 16. & CAC/GTG & & -2.088824 & 17 & 32. & TCA/AGT & & -1.042342 & 19 \\
\hline
\end{tabular}

Estimated free energy parameters for unique DNA NN doublets and triplets and their corresponding counts of appearance in the perfect match data set. All parameters have been estimated using experimental values measured at $37^{\circ} \mathrm{C}$ and $1 \mathrm{M}$ sodium concentration.

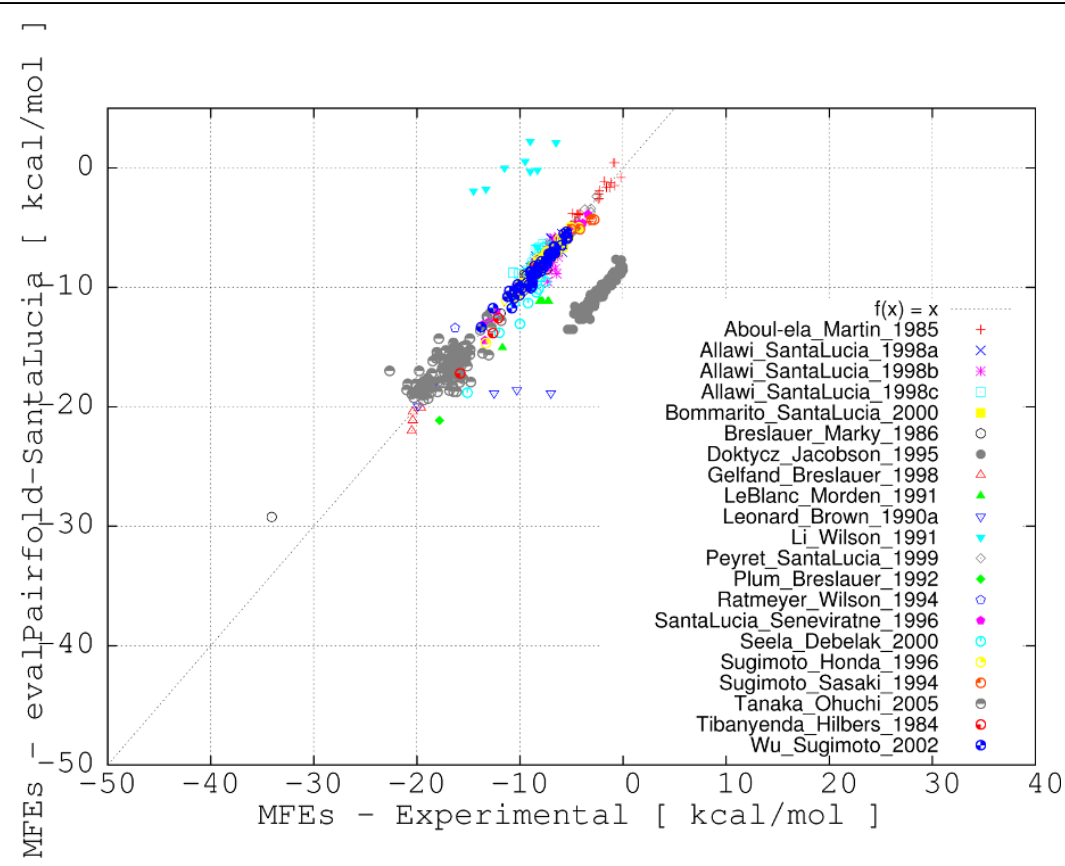

Figure 1 Correlation plot for the evaluation of free energy estimates (EVAL-FE) obtained with MultiRNAFold (with SantaLucia parameters) versus experimental free energies. The correlation of free energy estimates for all 695 DNA duplexes are represented. The plot depicts with different symbols and colors the source for each data point. 


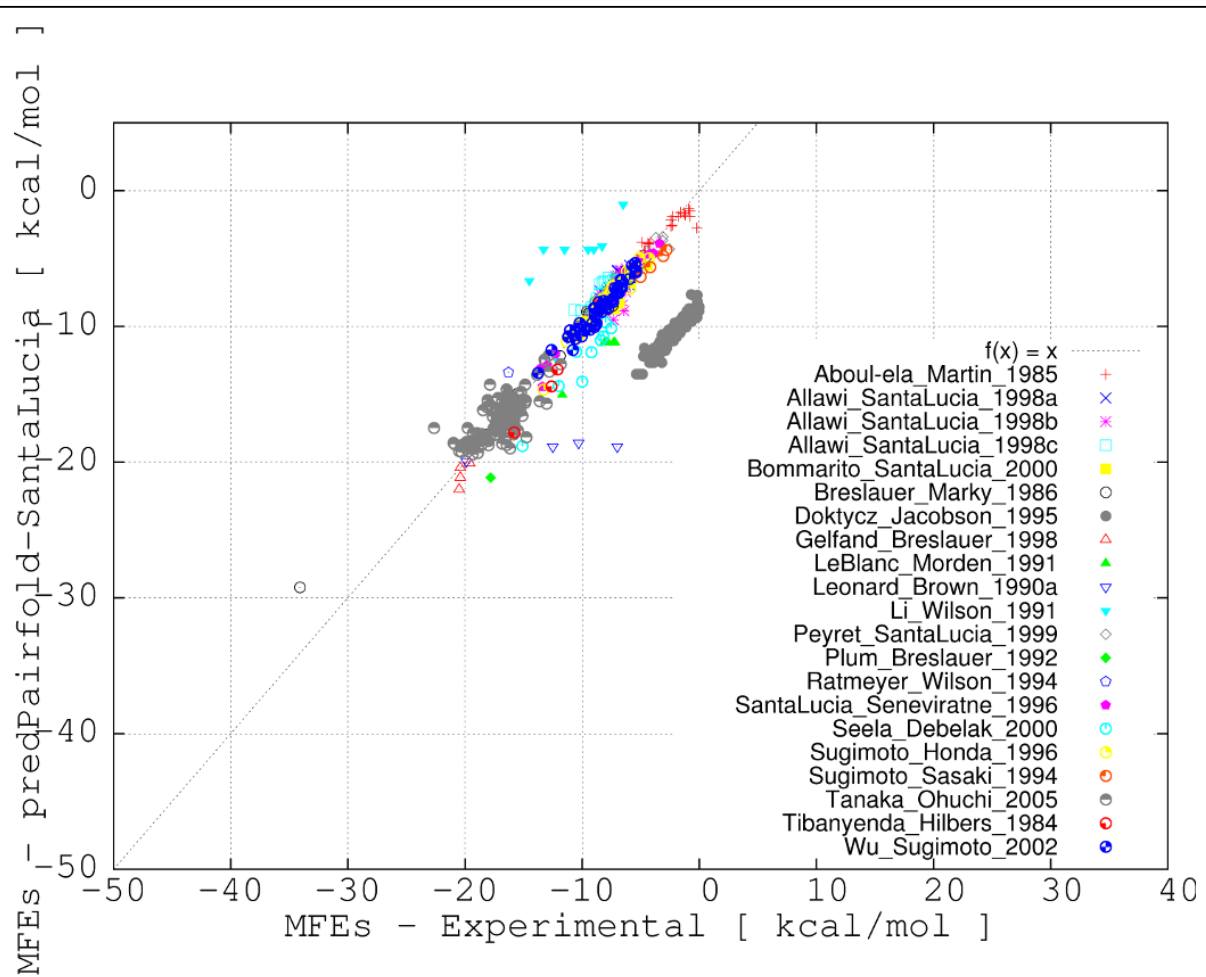

Figure 2 Correlation plot for the evaluation of secondary structure predictions (EVAL-SS) obtained with MultiRNAFold (with SantaLucia parameters) versus experimental free energies. The correlation of free energies for predicted secondary structures for all 695 DNA duplexes are represented. The plot depicts with different symbols and colors the source for each data point.

[25], Breslauer [26], Blake [27], Benight [28], SantaLucia [29], Sugimoto [30] and the Unified set [31]. Our set of $\mathrm{NN}$ thermodynamic doublet parameters summarized in Figure 12 differs from the unified parameters by less than $0.5 \mathrm{kcal} / \mathrm{mol}$ in 8 out of 10 cases. We also notice that our NN set follows in general the reported qualitative trend in order of decreasing stability: GC/CG = $\mathrm{CG} / \mathrm{GC}>\mathrm{GG} / \mathrm{CC}>\mathrm{CA} / \mathrm{GT}=\mathrm{GT} / \mathrm{CA}=\mathrm{GA} / \mathrm{CT}=\mathrm{CT} /$ $\mathrm{GA}>\mathrm{AA} / \mathrm{TT}>\mathrm{AT} / \mathrm{TA}>\mathrm{TA} / \mathrm{AT}$ with one exception, namely GG/CC has a higher weight than GC/CG and CG/GC, an effect that could be caused by the low representation of the GG/CC doublets in the training set and by the absence of duplex initiation parameters in our model.

\section{Conclusions}

In this work we showed that no major differences exist among free energy estimations of short DNA duplex hybridization when comparing four publicly available programs that employ various sets of thermodynamic parameters.

Here we introduce a simplified TNN Model based on triplets interactions for perfect match hybridizations of DNA duplexes. The model is able to approximate free energies for DNA duplexes under various experimental conditions with higher accuracy and lower RMSEs compared to the four publicly available programs considered in this work. The improvement is more noticeable for DNA duplexes at non-standard experimental temperature conditions (for example at $25^{\circ} \mathrm{C}$ ). This improvement obtained with the TNN Model based on triplets could be explained by the presence of a larger set of parameters consisting of 32 unique triplets (compared to only 10 unique doublets in the classical TNN Model) that better capture the impact of sequence components on the overall free energy of a DNA duplex. An alternative and potential complementary explanation of these improvements is the use of a wider variety of experimental data points in the thermodynamic parameter extrapolation process (the model training stage) compared to the smaller and less diverse data sets used in the other four programs. Nevertheless, we notice that additional experimental data employing longer and more diverse sequences is required in order to obtain a better approximation of free energies for DNA duplexes at other non-standard experimental conditions.

Three extensions of the TNN-Triplets-PM Model might improve its performance, given that additional experimental data that covers a higher percentage of the parameters and experimental condition combinations is obtained experimentally: (i) the model can incorporate 

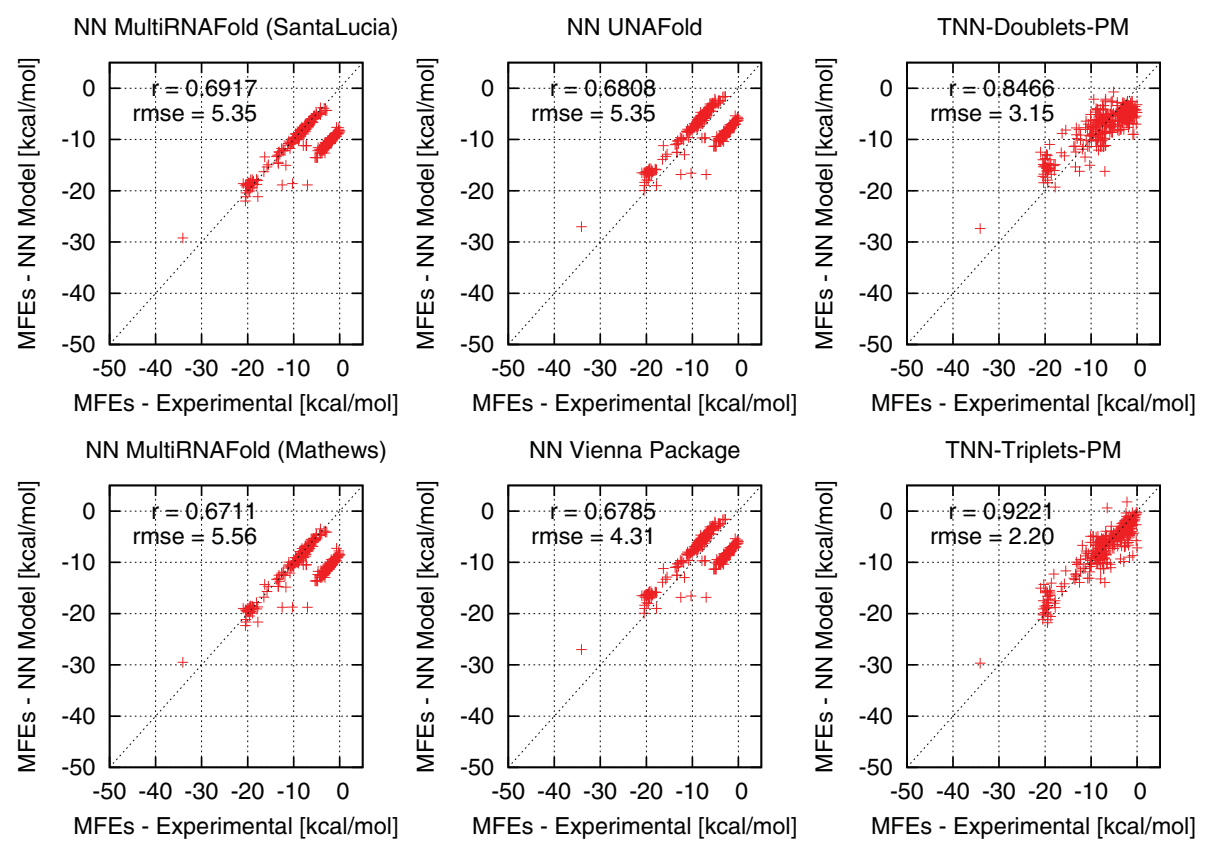

Figure 3 Correlation plots for estimated versus experimental free energies of perfect matches. Each correlation plot consists of 340 data points corresponding to all perfect match duplexes covering all temperatures, sequence and sodium concentrations. The top left plot depicts the correlation between experimental free energies and free energies estimated by MultiRNAFold with SantaLucia parameters. The Pearson correlation equals 0.6917 and the RMSE is 5.35. The bottom left plot depicts the correlation between experimental free energies and free energies estimated by MultiRNAFold with Mathews parameters. The Pearson correlation equals 0.6711 and the RMSE is 5.56. The top middle plot depicts the correlation between experimental free energies and free energies estimated by UNAFold. The Pearson correlation equals 0.6808 and the RMSE is 5.35. The bottom middle plot depicts the correlation between experimental free energies and free energies estimated by Vienna Package. The Pearson correlation equals 0.6785 and the RMSE is 4.31. The top right plot depicts the correlation between experimental free energies and free energies estimated by the TNN-Doublets-PM Model. The Pearson correlation equals 0.8466 and the RMSE is 3.15. The bottom right plot depicts the correlation between experimental free energies and free energies estimated by the TNN-Triplets-PM Model. The Pearson correlation equals 0.9221 and the RMSE is 2.20 .

weighted additive terms that account for hybridization initialization, temperature, $\mathrm{pH}$, sodium concentration or sequence concentrations; (ii) the model can incorporate symmetrical and asymmetrical internal loops, multibranch loops, dangling ends and hairpin rules similar to those already existent in the classical TNN Model; (iii) the model can also incorporate positional dependencies of triplets with respect to the 5' and 3' ends of the sequences.

\section{Methods}

The present study is divided into two major sections:

- Evaluation of free energy estimates (EVAL-FE): a comparative assessment of free energies calculated for DNA duplexes using different methods when both the duplex sequence and the duplex experimental secondary structure are given.

- Evaluation of secondary structure predictions (EVAL-SS): an accuracy assessment of secondary structure predictions when only the duplex sequence is given and the secondary structure is predicted.

\section{Table 5 Model training}

Require: $A$ thermodynamic model $T$, an input set $S$ with perfect match DNA duplexes.

Ensure: An optimal set of thermodynamic DNA parameters $X$ for the input model

1: Initialize counts matrix $F$ with zeros for all unique doublets/triplets

2: Initialize results matrix $R$ with experimentally approximated free energies for each duplex

3: for $i=0$ to $\|S\|$ do

4: Count unique doublets/triplets in duplex S[i] and update $F$

5: end for

6: Solve the equation $X=\arg \min _{X}(F \times X-R)^{2}$

7: return $X$

\section{Data}

The benchmark data set used in this work consists of 695 experimental free energies and secondary structures for DNA duplexes, including 340 perfect matches and 355 imperfect matches. We collected these data from 29 publications and we present its characteristics in Table 1. We must mention that a total of 42 DNA duplexes were removed from the original data set (with 737 DNA 


\section{Table 6 Model evaluation}

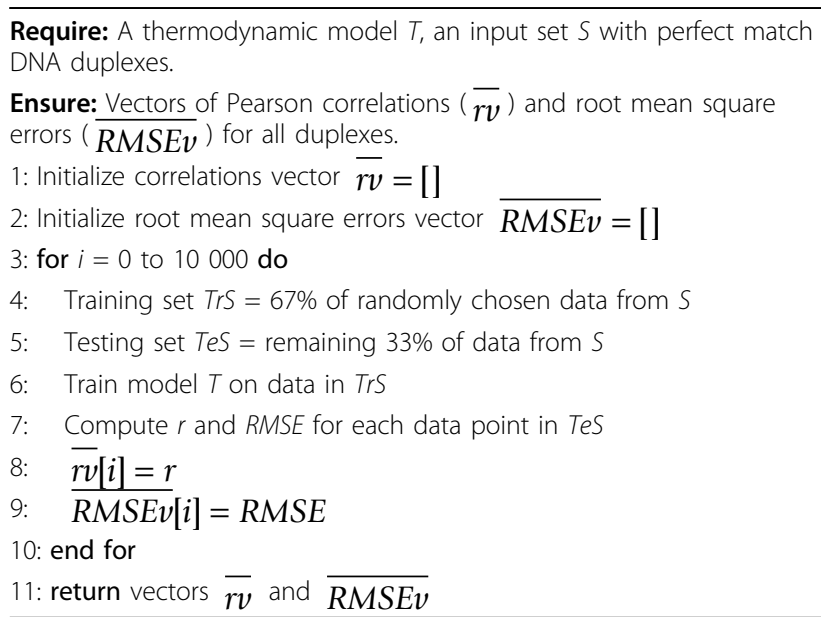

duplexes - see Additional file 1) because the ctEnergy function from UNAFold failed to produce valid free energies, due to the lack of DNA parameters for mismatches. The removed data corresponds to 30 duplexes from [31], 4 duplexes from [32], 4 duplexes from [33], 2 duplexes from [34] and 2 duplexes from [35]. The lengths of DNA sequences in the data set range from 4 nucleotides [29] to 30 nucleotides [36], some of them (length 8 and 9) being over represented (see Figure 13).

The GC-content (\%) of the sequences in the benchmark data set (see Figure 14) cover the whole spectra from $0 \%$ to $100 \%$, with a dominant peak at $50 \%$.

Sequence concentrations range from $17.5 \times 10^{-6} \mathrm{M}$ in [33] to $10^{-4} \mathrm{M}$ in $[20,21,31,37,38]$. The sodium concentration varies from $0.1 \mathrm{M}$ in [39] and [40] to $1 \mathrm{M}$ in 20 out of 29 sources. The reported free energies were measured at reaction temperatures ranging between $24.85^{\circ} \mathrm{C}$ $[33,41]$ and $50^{\circ} \mathrm{C}[32]$.

\section{Free energy calculations}

In this study, three publicly available packages were used to calculate and compare the free energies for pairs of short DNA sequences: MultiRNAFold (with Mathews and SantaLucia parameters), UNAFold and the Vienna Package. All packages implement the TNN Model based on base doublet parameters.

The basic free energy calculations implemented in MultiRNAFold and Vienna Package are performed according to the Gibbs equation:

$$
\Delta G_{T}^{o}=\Delta H^{o}-T \cdot \Delta S^{o}
$$

where $G^{\circ}$ is the free energy measured, $H^{\circ}$ is the enthalpy, $T$ is the absolute temperature measured in degrees Kelvin and $S^{\circ}$ is the entropy.
For a general two-state transition process of the type $A+B \rightleftharpoons A B$ at equilibrium, the free energy change is calculated as follows:

$$
\Delta G^{o}=-R \cdot T \cdot \ln (k)
$$

where $R$ is the gas constant $(1.98717 \mathrm{cal} /(\mathrm{mol} \mathrm{K})), T$ is the absolute temperature, and $k$ is the equilibrium constant.

\section{Computational procedures}

The two main sections of this study, namely the evaluation of free energy estimates and the evaluation of secondary structure predictions, employ computational procedures made available in the corresponding software packages. The evaluation of free energy estimates (EVAL-FE) includes the following procedures:

- The function free energy pairfold (sequence1, sequence2, known structure) is provided by the MultiRNAFold package to compute the free energy for two sequences when the known secondary structure is given. The pairfold wrapper has been slightly modified to accept as parameters: two sequences, the temperature, the set of parameters (Mathews or SantaLucia), the nucleic acid (DNA or RNA) and the type of hybridization (with or without intra-molecular interactions between nucleotides).

- The function RNAeval is provided by the Vienna Package to compute the free energy for two sequences when the known secondary structure is provided. We wrote a Python wrapper that calls this function with the following parameters: - T temperature, -P dna.par. The wrapper also pre-processes the sequence and structure input so to satisfy the interactivity requirements of the RNAeval function.

- The function ctEnergy is provided by the UNAFold Package to compute the free energy for two sequences when the known secondary structure is given. We wrote a Python wrapper that preprocesses the sequences and structures into a CTformatted input file and calls the function with the following parameters: $-n$ DNA, -t temperature, $-N$ sodium concentration.

The evaluation of secondary structure predictions (EVAL-SS) includes the following procedures:

- The function pairfold mfe (sequence1, sequence2, output structure) is provided by the MultiRNAFold package to compute the minimum free energy secondary structure for two DNA sequences that fold into 'output structure'. The pairfold wrapper has been slightly modified as described above. 


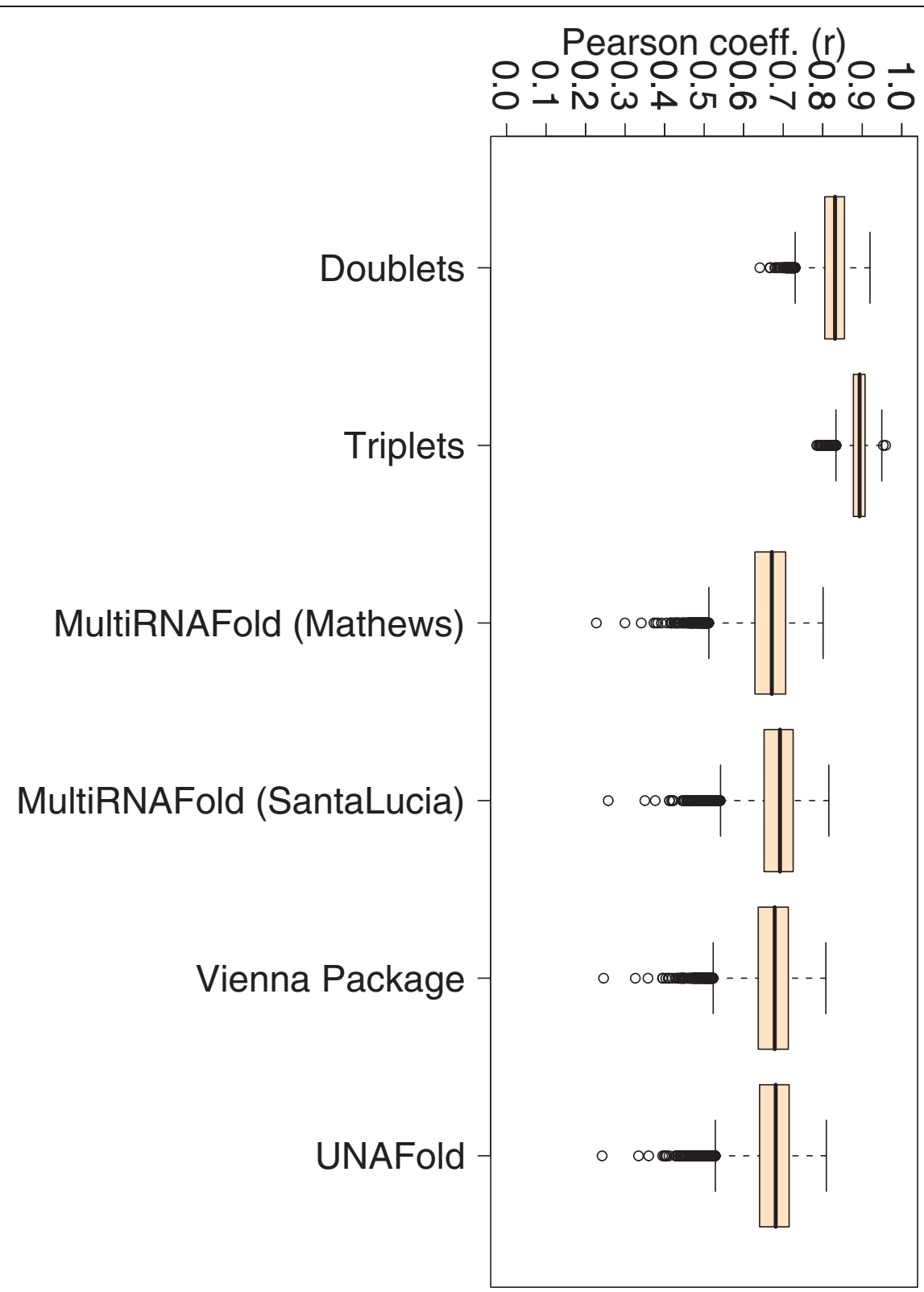

Figure 4 Box plots for Pearson correlations (r) corresponding to all $\mathbf{3 4 0}$ perfect match duplexes. The figure represents box plots for Pearson correlation coefficients for all 340 perfect match duplex free energies measured at various temperatures, sequence and sodium concentrations. The doublet- and triplet-based models were executed 10000 times on randomly selected subsets with $67 \%$ training data and $33 \%$ testing data.

- The function RNAcofold is provided by the Vienna Package to predict the free energy secondary structure for two sequences. A wrapper has been created for this function to accommodate the input and the parameters for the interactive interface as described above.

- The script UNAFold.pl is provided by the UNAFold Package to predict the free energy secondary structure for two sequences. We wrote a Python wrapper that pre-processes the sequences and structures into a CT-formatted input file and calls the function with the same parameters as for the ctEnergy function.

\section{The TNN-Triplets-PM Model}

For the case when only free energies for perfect matches are evaluated, we explore an approach that extends the classical TNN Model by looking at base triplets. A similar approach was introduced in 1999 by [16]. For the 


\section{RMSE}

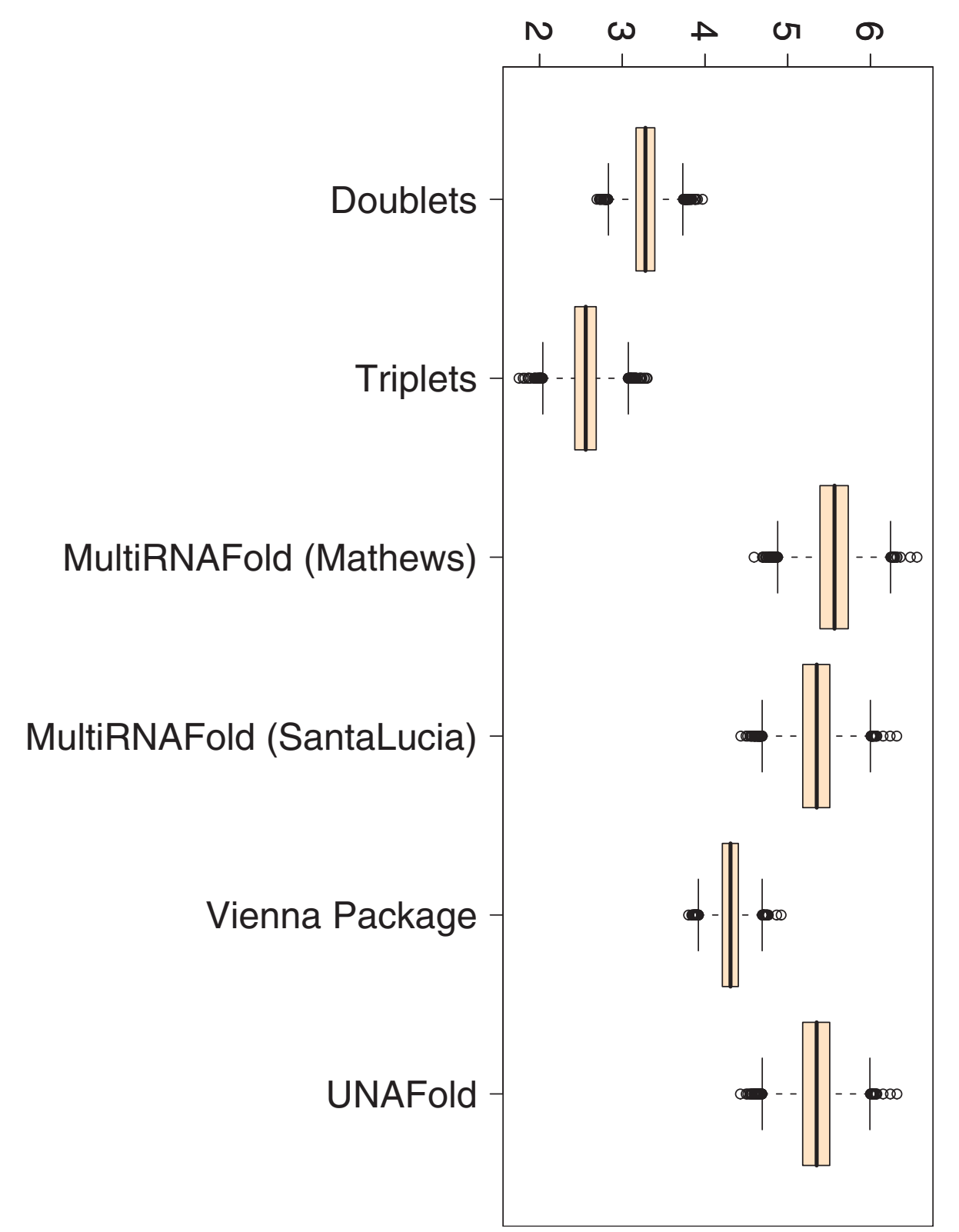

Figure 5 Box plots for RMSEs corresponding to all $\mathbf{3 4 0}$ perfect match duplexes. The figure represents box plots for RMSEs for all 340 perfect match duplex free energies measured at various temperatures, sequence and sodium concentrations. The doublet- and triplet-based models were executed 10000 times on randomly selected subsets with 67\% training data and 33\% testing data.

classical TNN Model, only ten different nearest-neighbour interactions (out of 16) are possible for any Watson-Crick DNA duplex structure due to rotational identities. Here $\mathrm{A}$ is hydrogen bonded with $\mathrm{T}$ and $\mathrm{G}$ is hydrogen bonded with $\mathrm{C}$. These interactions are AA/ TT, AT/TA, TA/AT, CA/GT, GT/CA, CT/GA, GA/CT, CG/GC, GC/CG, and GG/CC. Here the slash, /, separates strands in anti parallel orientation (e.g., TC/AG means 5' - TC - 3' paired with 3' - AG - 5'). While the classical TNN model assumes that the stability of a DNA duplex depends on the identity and orientation of only close neighbouring base pairs, the one based on triplet interactions takes the approach one step further and assumes that the stability of a DNA duplex can be approximated if the first two neighbours of each base are considered. Since our goal is to examine and 


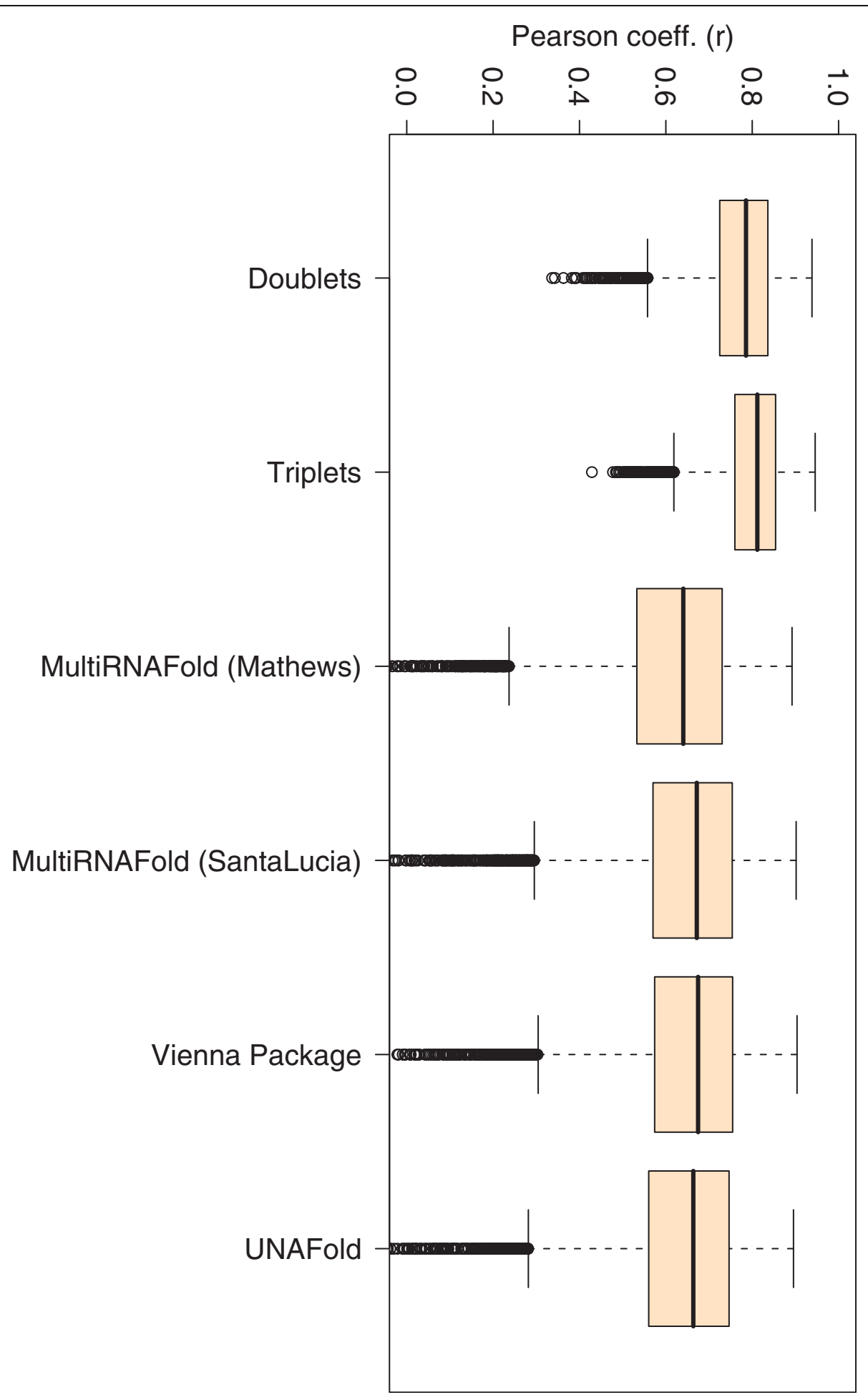

Figure 6 Box plots for Pearson correlations (r) corresponding to 197 perfect match duplex free energies measured at $25^{\circ} \mathrm{C}$. The figure represents box plots for Pearson correlation coefficients for 197 perfect match duplex free energies measured at $25^{\circ} \mathrm{C}$ and a sodium concentration of $1 \mathrm{M}$. The doublet- and triplet-based models were executed 10000 times on randomly selected subsets with $67 \%$ training data and $33 \%$ testing data. 


\section{RMSE}

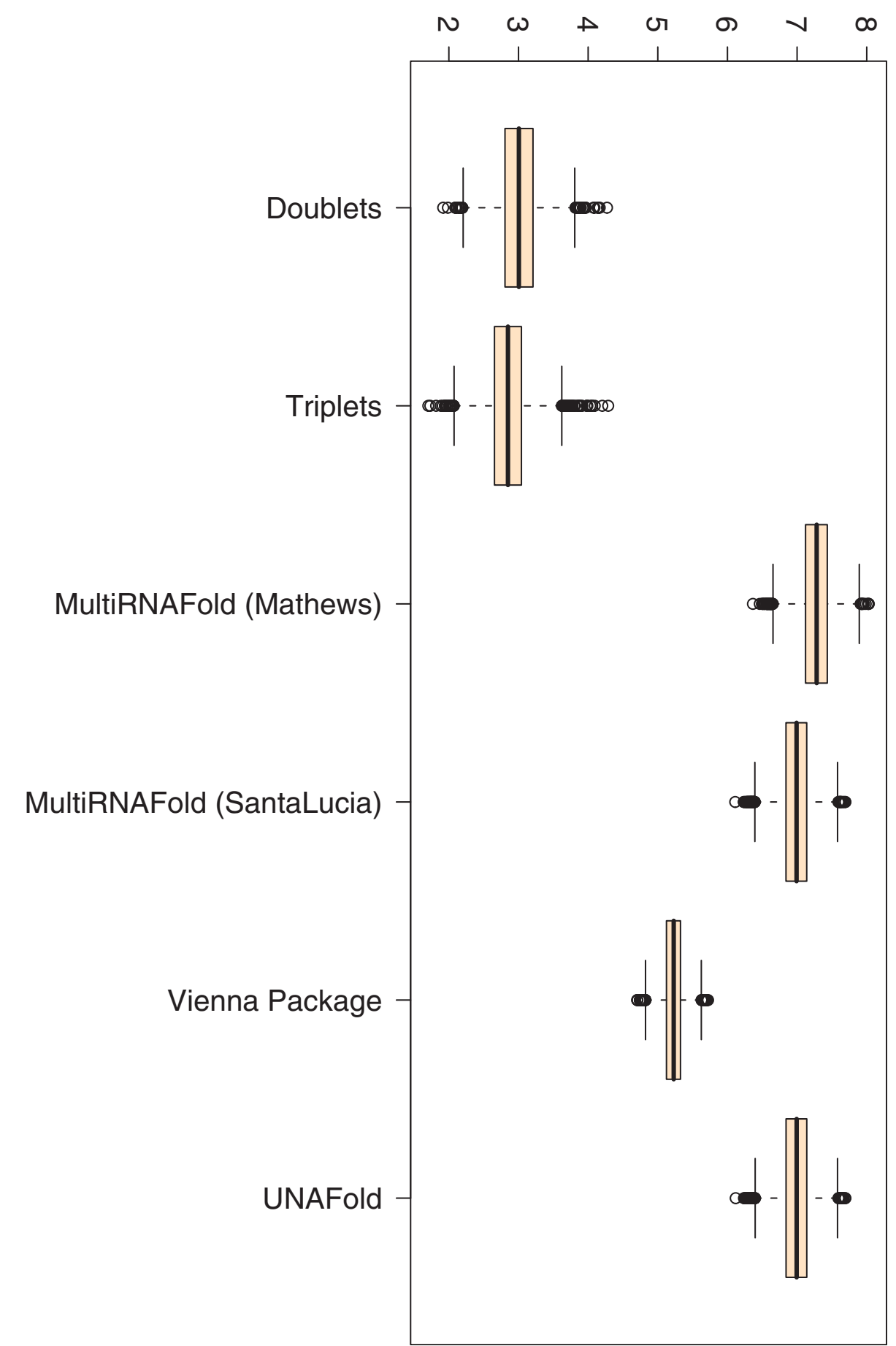

Figure 7 Box plots for RMSEs corresponding to 197 perfect match duplex free energies measured at $25^{\circ} \mathrm{C}$. The figure represents box plots for RMSEs for 197 perfect match duplex free energies measured at $25^{\circ} \mathrm{C}$ and a sodium concentration of $1 \mathrm{M}$. The doublet- and tripletbased models were executed 10000 times on randomly selected subsets with 67\% training data and 33\% testing data. 


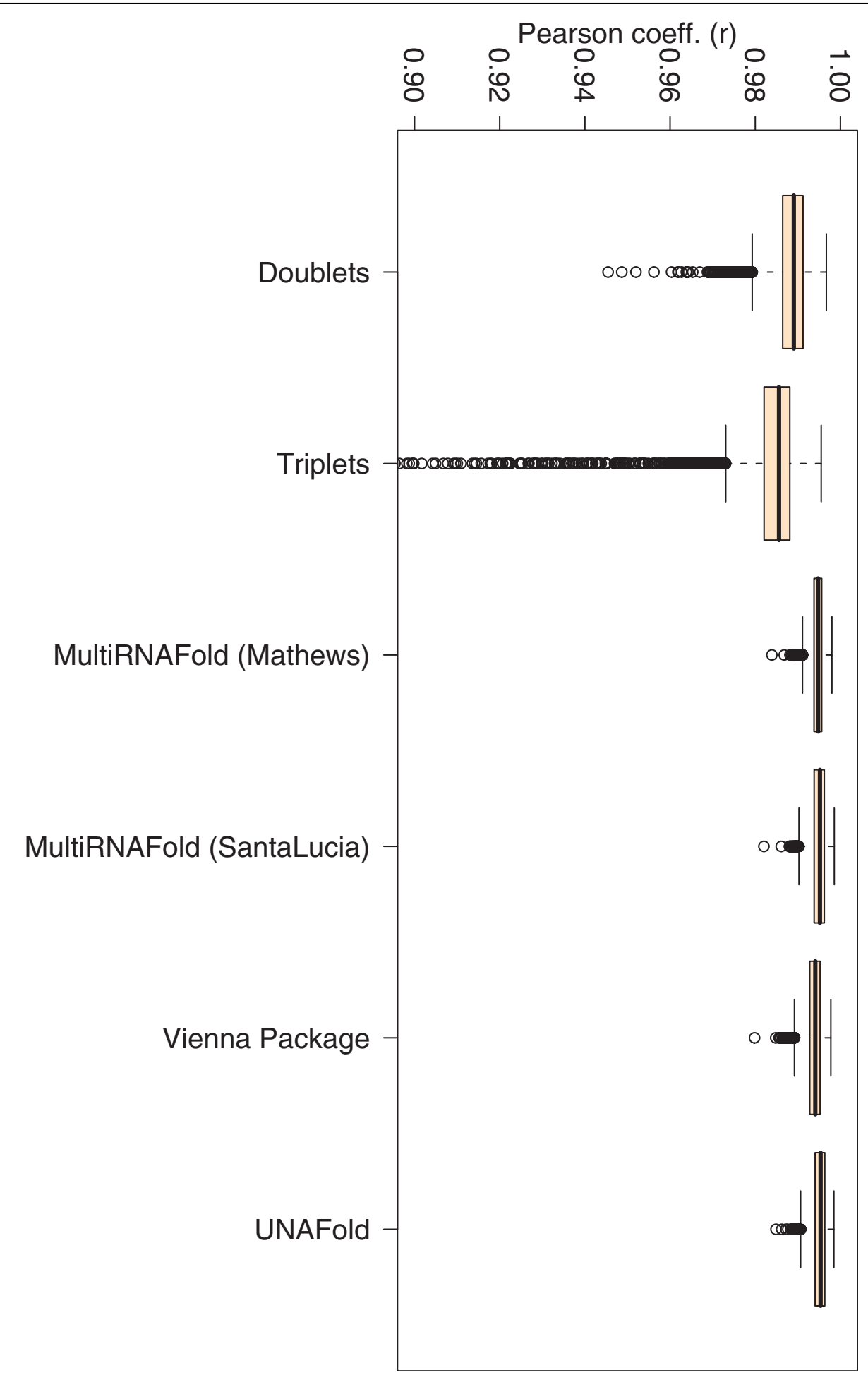

Figure 8 Box plots for Pearson correlations (r) corresponding to 143 perfect match duplex free energies measured at $37^{\circ} \mathrm{C}$. The figure represents box plots for Pearson correlation coefficients for 143 perfect match duplex free energies measured at $37^{\circ} \mathrm{C}$ and a sodium concentration of $1 \mathrm{M}$. The doublet- and triplet-based models were executed 10000 times on randomly selected subsets with $67 \%$ training data and $33 \%$ testing data. 


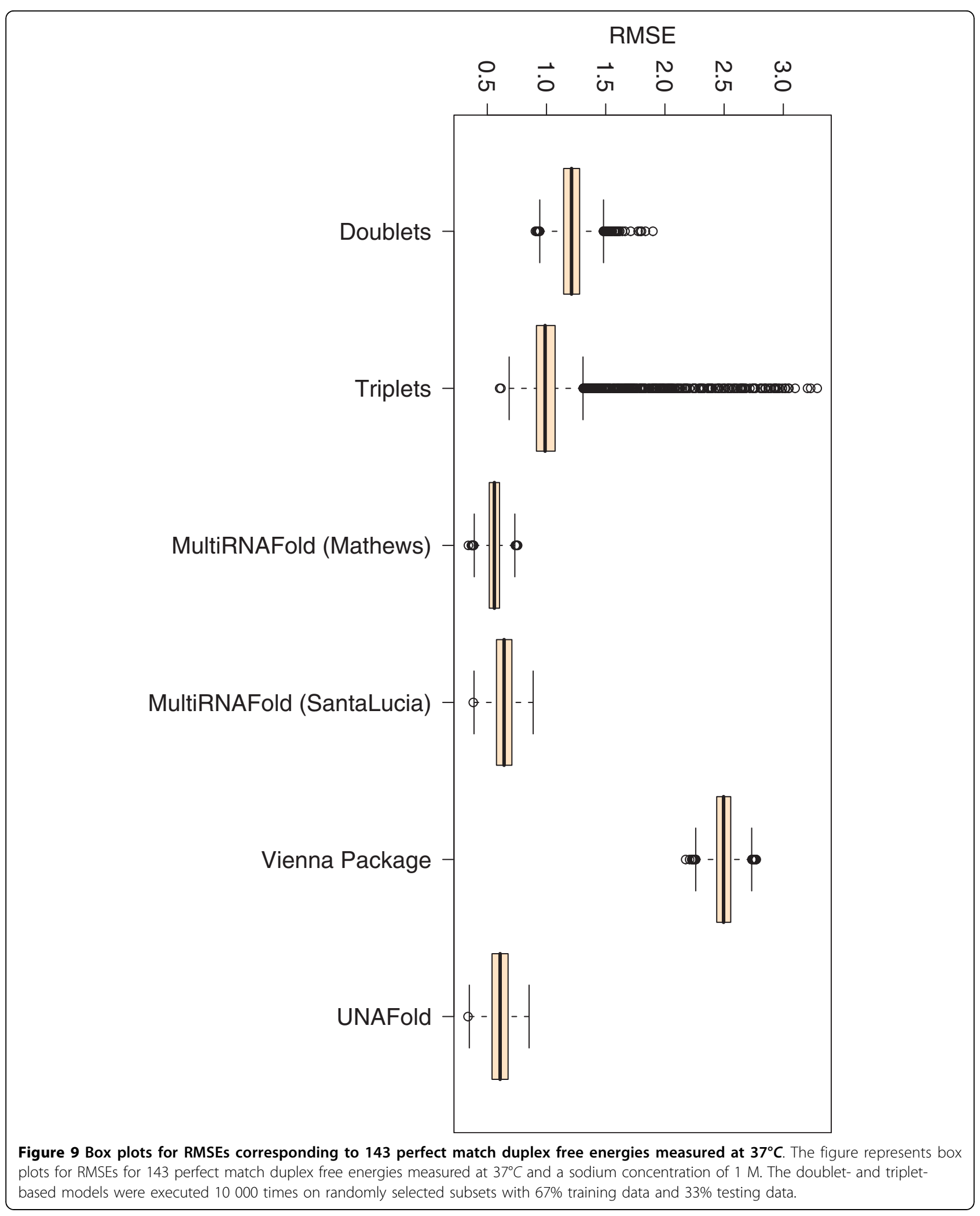




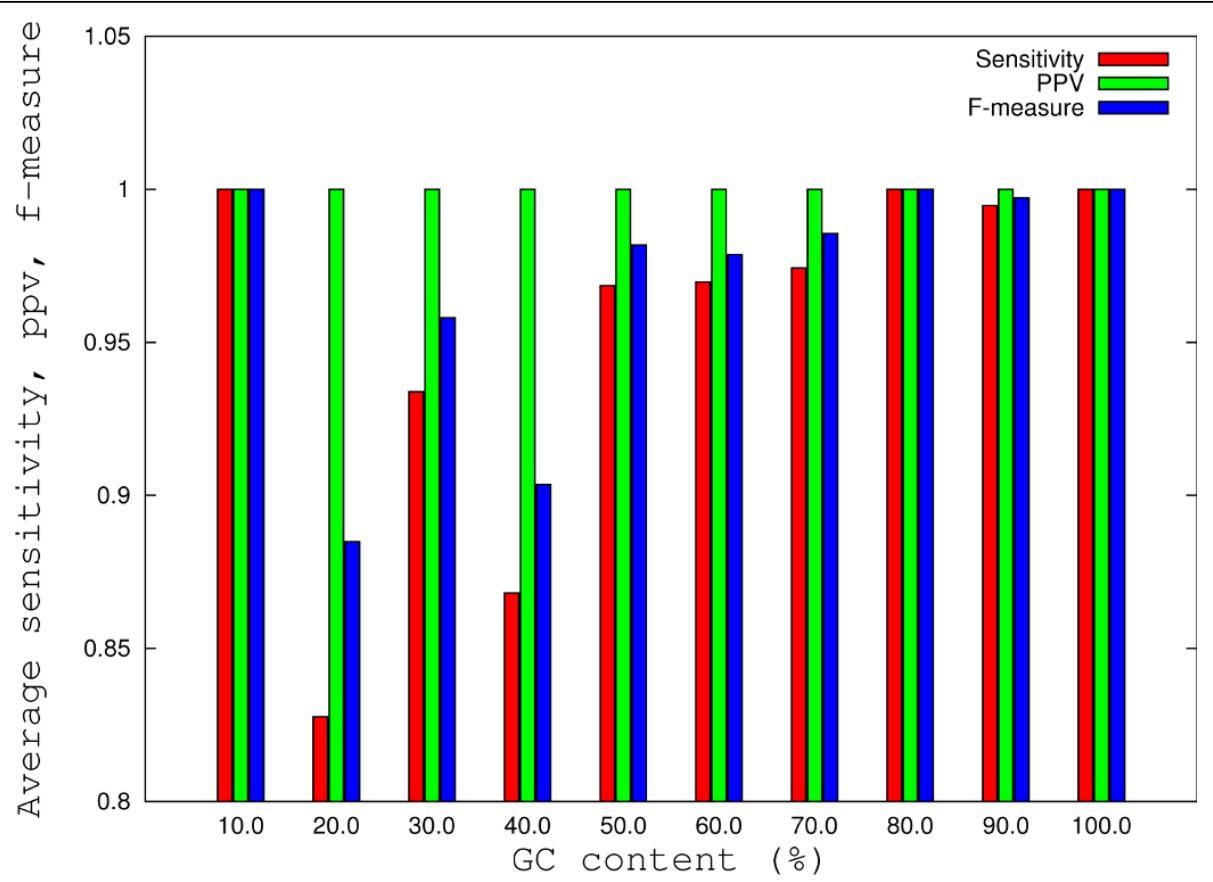

Figure 10 Histograms of average variations of the SENS, PPV and F-measure with respect to sequence length. The histograms corresponding to average variations of the SENS, PPV and F-measure (defined in Methods) with respect to sequence length were calculated for all 695 duplexes. All minimum free energies were calculated with PairFold-SantaLucia.

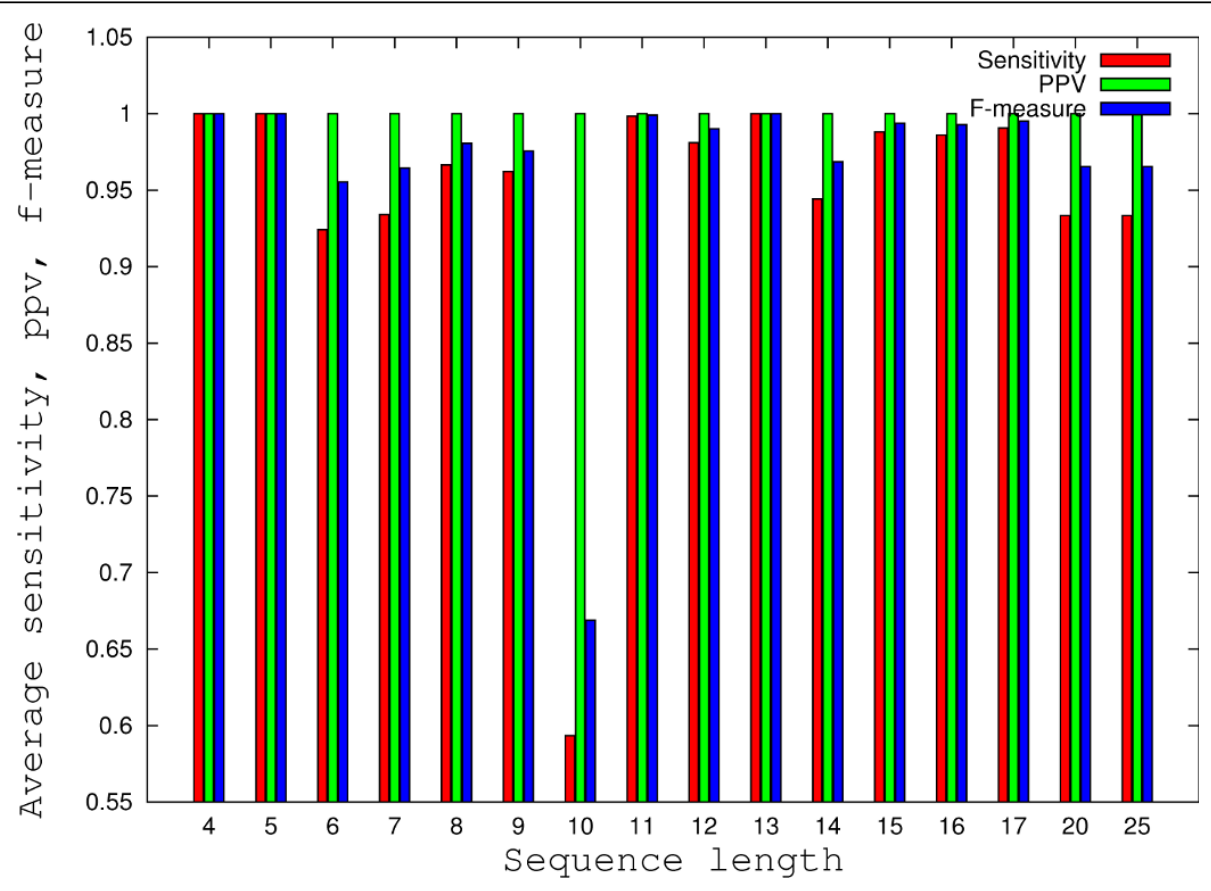

Figure 11 Histograms of average variations of the SENS, PPV and F-measure with respect to GC-content percentage. The histograms corresponding to average variations of the SENS, PPV and F-measure (defined in Methods) with respect to GC-content percentage were calculated for all 695 duplexes. All minimum free energies were calculated with PairFold-SantaLucia. 


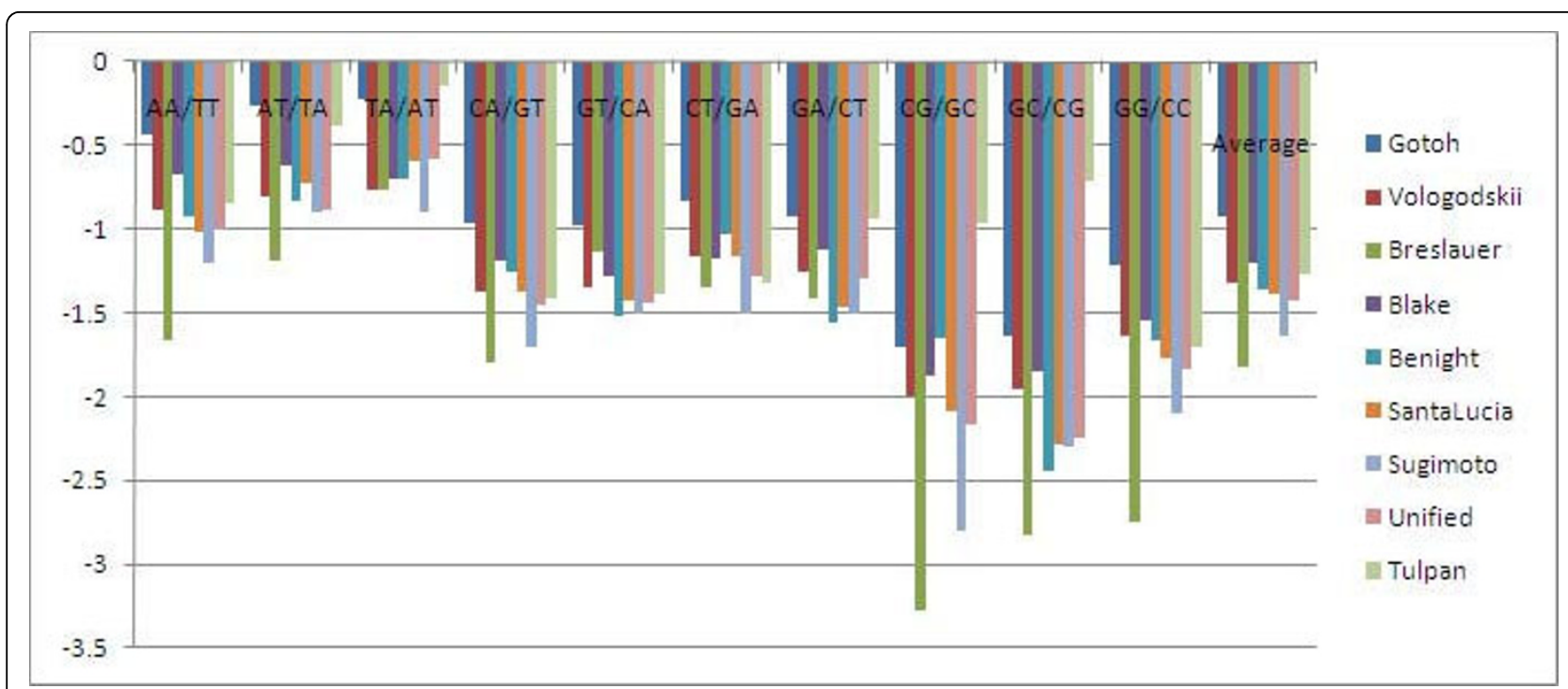

Figure 12 Variation of doublet NN values for $\mathbf{9}$ sets of parameters. Free energy values corresponding to nine sets (our set and 8 others) of thermodynamic nearest-neighbour doublet parameters at $37^{\circ} \mathrm{C}$ are displayed in this plot. Four (Gotoh, Vologodskii, Blake and Benight) out of the eight publicly available sets of doublet parameters correspond to models that do not account for initiation penalties for duplex formations [18], and the sodium concentration for their experiments was between $0.0195 \mathrm{M}$ and $0.195 \mathrm{M}$. For the other 4 sets (Breslauer, SantaLucia, Sugimoto and Unified) the sodium concentration equals $1 \mathrm{M}$.

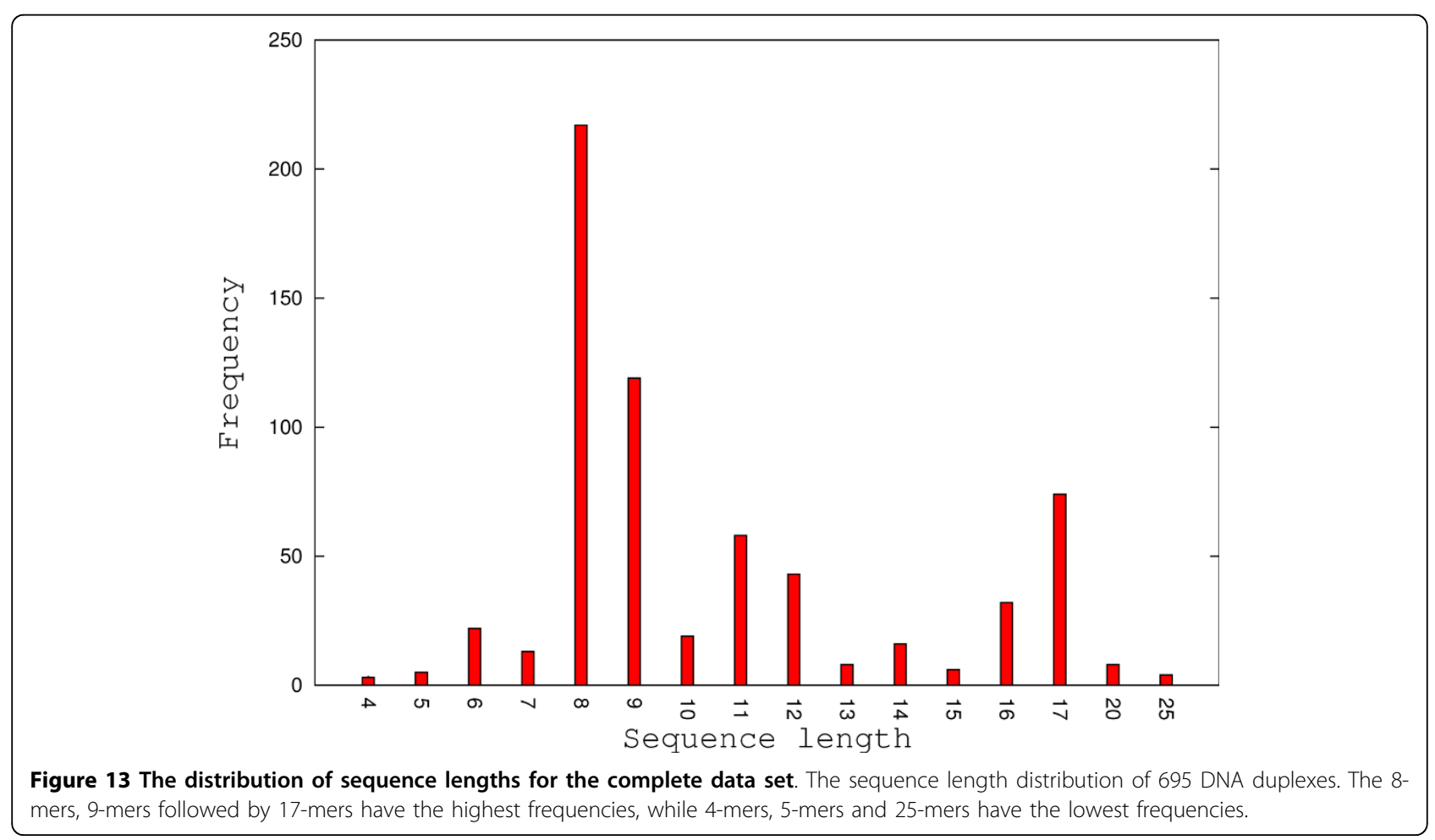




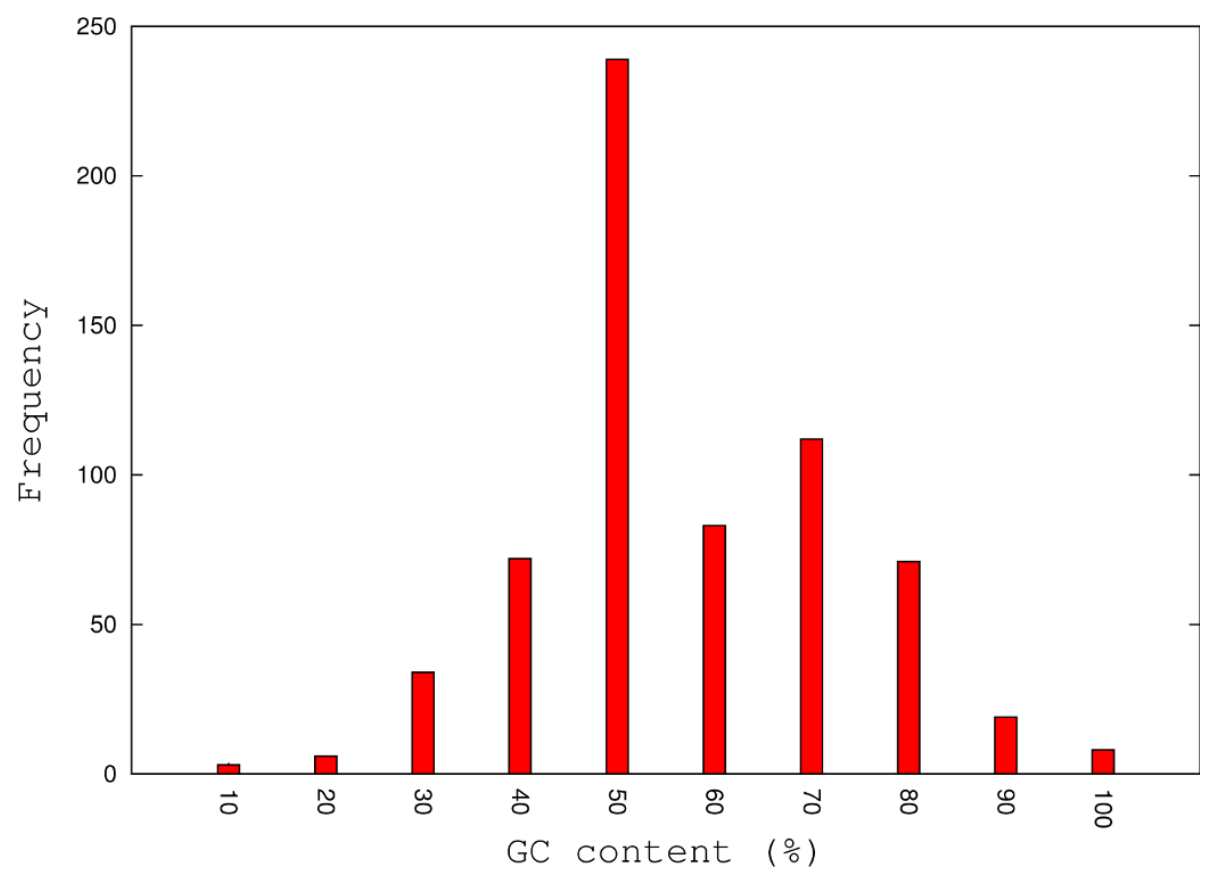

Figure 14 The distribution of GC-content percentages for the complete data set. The distribution of GC-content percentages for 695 duplexes. The majority of sequences have a 50\% GC-content while only a few sequences have either low (10\%, 20\%) or high (100\%) GC-content percentages.

compare the impact of doublet versus triplet interactions on the accuracy of free energy estimations, the approach proposed in this paper relies solely on triplet interactions, while the one proposed by [16] uses a more complex cumulative approach that combines singlet, doublet and triplet interactions within the same model. Due to rotational identities, only 32 different nearest-neighbour interactions are possible (out of a total of 64) for any Watson-Crick triplet structure. These interactions are enumerated in Table 2 together with corresponding parametric values obtained via a least-mean squared optimization solution for equation 3.

$$
F \times X=R
$$

where $F$ is a $N \times 32$ matrix of counts for all perfect match data points, $X$ is a vector with 32 unknown triplet parameter values, and $R$ is a vector with $N$ free energy experimental values for perfect matches. We solve the following equation:

$$
X=\arg \min _{X}(F \times X-R)^{2}
$$

These values were obtained by using an over determined system of $N$ equations (3) and solving equation 4 with a least-mean squared optimization function (implemented in the backslash operator for matrices) available in Matlab 7.7. Here $N$ takes the value 228 (67\% of 340 perfect match free energies measured at $25^{\circ} \mathrm{C}$ and $37^{\circ} \mathrm{C}$ ), 132 (67\% from 197 perfect match free energies measured at $\left.25^{\circ} \mathrm{C}\right)$, or $96(67 \%$ of 145 perfect match free energies measured at $37^{\circ} \mathrm{C}$ ). The system with $N$ equations has been extrapolated by selecting from the initial data set only the free energy measurements for perfect match DNA duplexes and counting the frequency of triplets in each duplex. Thus, for each duplex, the sum of parametric values for each triplet multiplied with its counts equals the experimental free energy. While our model is very simple and currently does not take into consideration mismatches, internal loops, and dangling ends, its strength is given by its ability to estimate perfect match DNA duplex free energies for a wide range of sodium, sequence and target concentrations and temperatures. This strength is given by the presence of a large and mixed training data set that was used to extrapolate the nearest-neighbour $(\mathrm{NN})$ parameters for both the doublet- and the triplet-based models.

\section{Model training and testing}

The training process for the TNN-Triplets-PM Model is summarized in Table 5. We first process the input set, which contains perfect match DNA sequences and their corresponding experimentally derived free energies. The processing consists of scanning each perfect match sequence from left to right by moving a window of size 3 nucleotides (or 2 for the doublets) and counting the 
frequency of each of the 32 unique triplets. We record each frequency at corresponding positions $(i, j)$ in matrix $F$ and each experimentally derived free energy is recorded at position $i$ in matrix $R$. Here $i$ represents the number of the sequence in the set and $j$ represents the number of the triplet (from 1 to 32), whose frequency is recorded. After matrices $F$ and $R$ have been populated, a solution for equation 4 is computed and the value of vector $X$ containing free energy parameters for all the unique triplets is reported.

The evaluation process of the TNN-Triplets-PM Model is summarized in Table 6. The evaluation process is repeated 10000 times in this work. Each iteration consists of the following steps. First the data set is divided uniformly at random in a training set, $\operatorname{Tr} S$ consisting of $67 \%$ of the data and a testing set, TeS that contains the remaining 33\%. Next, the training process described in Table 5 is used to extrapolate the first set of perfect match triplet parameters. The derived parameters are used next to compute the Pearson momentum correlation coefficients and the RMSEs for each DNA perfect match duplex from TeS. Each correlation coefficient and RMSE is recorded in corresponding vectors to be analyzed later. The complete coverage of the triplet space, i.e. all possible triplets during the generation of training and testing sets using a randomized mechanism is not ensured for some of the 10000 sets mostly due to the presence of a few under-represented (less than $20 \mathrm{CCC} / \mathrm{GGG}$ ) or over-represented (more than $180 \mathrm{GAC} / \mathrm{CTG}$ ) triplets that characterize the data set with perfect matches (see Figures 15 and 16). Nevertheless, we noticed that the training sets that produced

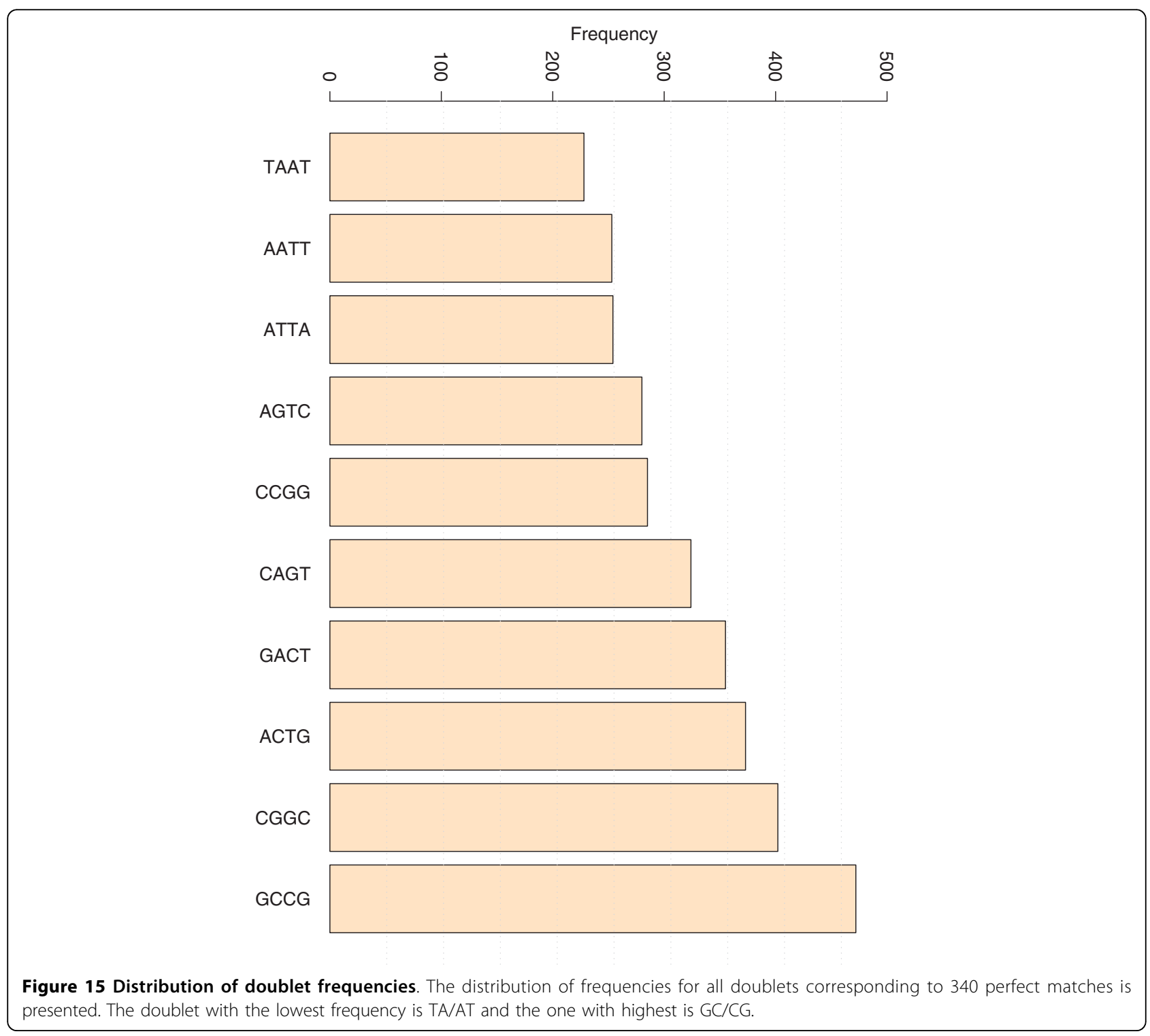




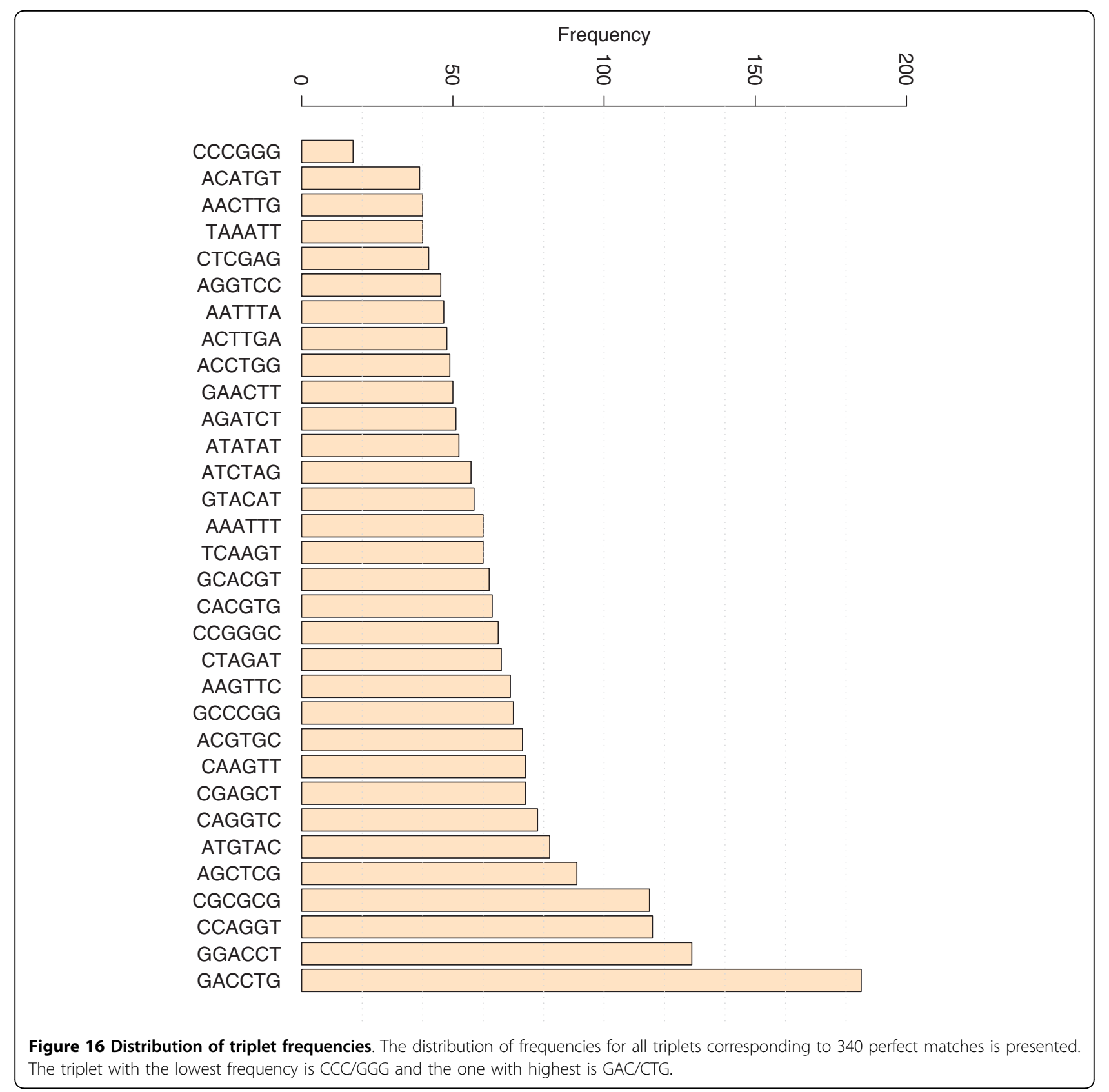

the best results cover completely the triplet space. The same coverage was observed for the doublets.

\section{Comparative measures}

We use a large number of measures of similarity between experimental and computed free energies. Some of these measures were previously used by [42] to compare melting temperatures obtained with different methods and by [6] to estimate model parameters for RNA secondary structure prediction. If not stated otherwise, all comparisons in this paper were done on a data set comprising 695 pairs of DNA sequences collected from 29 publications. The measures used in this study are grouped in two categories, namely:

\section{Measures that evaluate accuracy of free energy estimations}

The following measures are used for free energy estimations of the known structures, as well as free energy estimations of predicted structures.

- the observed absolute difference between experimental and estimated free energies (MFE_AD),

- the Pearson correlation coefficient (r), 
- the root mean squared error (RMSE),

\section{Measures that evaluate accuracy of secondary structure predictions}

- the secondary structure similarity index of experimental and predicted secondary structures (SSSI)

- the prediction sensitivity for secondary structures (SENS)

- the positive predictive value for secondary structures (PPV)

- the F-measure for predicted secondary structures (F)

For MFE_AD, SSSI, SENS, PPV and F we report the minimum, the first quartile, the median, the mean, the third quartile, the maximum and the standard deviation.

We define the secondary structure similarity index (SSSI) for two equally long structures as follows:

$$
S S S I=\frac{S S\left(s 1_{\exp }, s 1_{c a l c}\right)+S S\left(s 2_{\exp }, s 2_{c a l c}\right)}{\operatorname{len}\left(s 1_{\exp }\right)+\operatorname{len}\left(s 2_{\exp }\right)} \cdot 100
$$

where $s 1_{\text {exp }}, s 2_{\text {exp }}$ are two equally long structures obtained experimentally, $s 1_{\text {calc }}, s 2_{\text {calc }}$ are two equally long calculated structures, and $S S(a, b)$ is the total number of identical characters at corresponding positions in both structures. SSSI represents the percentage of positions in which two structures agree.

Unlike similar measures that assign a +1 score for two identical base pairs in two duplex structures, SSSI assigns $\mathrm{a}+1$ score for two base pairs that have either the start or the end positions identical. This mechanism allows the differentiation between duplex secondary structures that have either one (score +1$)$ or both (score +2 ) bases in a base pair correctly predicted.

The sensitivity, positive predictive value and F-measure are defined as in [6], namely:

$$
\begin{aligned}
& \text { Sensitivity }=\frac{\text { number of correctly predicted base pairs }}{\text { number of true base pairs }} \\
& P P V=\frac{\text { number of correctly predicted base pairs }}{\text { number of predicted base pairs }} \\
& \text { F-measure }=\frac{2 \cdot \text { Sensitivity } P P V}{\text { Sensitivity }+P P V}
\end{aligned}
$$

\section{Computational infrastructure}

The entire analysis of this study was done with $\mathrm{R}$ version 2.5.1, Perl 5.8.8 and Python 2.5. All computations were carried out on a Open SuSe 10.2 Linux (kernel version 2.6.18.2) machine equipped with a Pentium 4, $2.8 \mathrm{GHz}$ processor with $1 \mathrm{~GB}$ of RAM.

Additional file 1: Data set in comma separated value. The file contains information representing the data set used in this work. The data is structured on 15 columns as follows: (col 1) first sequence of the duplex, (col 2) second sequence of the duplex, (col 3) unique duplex ID containing the first and last authors of the papers that have first published the data, ( $\mathrm{Col} 4)$ dot-parenthesis notation of the secondary structure representation for the first sequence, (col 5) dot-parenthesis notation of the secondary structure representation for the second sequence, (col 6) experimental free energy measurement, (col 7) measurement error for the free energy, (col 8) experimental entropy measurement, (col 9) measurement error for the entropy, (col 10) experimental enthalpy measurement, (col 11) measurement error for the enthalpy, (col 12) experimental temperature of hybridization, (col 13) concentration for self-complementary sequences, (col 14) concentration for non self-complementary sequences, $(\mathrm{col} 15)\left[\mathrm{N} \mathrm{a}{ }^{+}\right.$concentration. Click here for file

[http://www.biomedcentral.com/content/supplementary/1471-2105-11105-S1.CSV]

\section{Acknowledgements}

Funding for this work was provided to DT and SL by the National Research Council of Canada. We gratefully acknowledge the helpful comments and suggestions provided by Dr. Anne Condon and Dr. Miroslava Cuperlovic-Culf. We thank Dr. Fazel Famili, Georges Corriveau and Natalie Hartford for proofreading our article and the anonymous reviewers of this manuscript for their valuable feedback.

\section{Author details}

${ }^{1}$ National Research Council of Canada, Institute of Information Technology, 100 des Aboiteaux Street, Suite 1100, Moncton, NB, E1A 7R1, Canada. 2Department of Genome Sciences, University of Washington, 1705 NE Pacific St, Seattle, WA 98195-5065, USA.

\section{Authors' contributions}

DT and MA planned the research, collected and curated the data, and wrote the paper. SL wrote the code for results computation and collection with UNAFold and the Vienna Package. DT wrote the code for results computation and collection with MultiRNAFold and analyzed the data. All authors read and approved the final manuscript.

\section{Received: 6 October 2009}

Accepted: 24 February 2010 Published: 24 February 2010

\section{References}

1. SantaLucia J, Turner DH: Measuring the thermodynamics of RNA secondary structure formation. Biopolymers 1997, 44(3):309-319.

2. Barbault F, Huynh-Dinh T, Paoletti J, Lanceloti G: A new peculiar DNA structure: NMR solution structure of a DNA kissing complex. J Biomol Struct Dyn 2002, 19(4):649-658.

3. Schmidt C, Welz R, Müller S: RNA double cleavage by a hairpin-derived twin ribozyme. Nucleic Acids Res 2000, 28(4):886-894.

4. Gharaibeh RZ, Fodor AA, Gibas CJ: Software note: using probe secondary structure information to enhance Affymetrix GeneChip background estimates. Comput Biol Chem 2007, 31(2):92-98.

5. Andronescu M, Zhang ZC, Condon A: Secondary structure prediction of interacting RNA molecules. J Mol Biol 2005, 345(5):987-1001.

6. Andronescu M, Condon A, Hoos HH, Mathews DH, Murphy KP: Efficient parameter estimation for RNA secondary structure prediction. Bioinformatics 2007, 23(13):19-28.

7. Hofacker IL: Vienna RNA secondary structure server. Nucleic Acids Res 2003, 31(13):3429-3431.

8. Markham NR, Zuker M: DINAMelt web server for nucleic acid melting prediction. Nucleic Acids Res 2005, , 33 Web Server: 577-581. 
9. Andronescu M: Algorithms for predicting the secondary structure of pairs and combinatorial sets of nucleic acid strands. PhD thesis University of British Columbia, Computer Science Department 2003.

10. Turner Lab, last visited: November 2008. http://rna.chem.rochester.edu/.

11. Mathews Lab Webpage, last visited: November 2008. http://rna.urmc. rochester.edu/.

12. SantaLucia Lab, last visited: November 2008. http://ozone3.chem.wayne. edu/home/.

13. Zuker $M$, Stiegler $P$ : Optimal computer folding of large RNA sequences using thermodynamics and auxiliary information. Nucleic Acids Res 1981, 9:133-148.

14. MCCaskill JS: The equilibrium partition function and base pair binding probabilities for RNA secondary structure. Biopolymers 1990, 29(67):1105-1119.

15. Wuchty S, Fontana W, Hofacker IL, Schuster P: Complete suboptimal folding of RNA and the stability of secondary structures. Biopolymers 1999, 49(2):145-165.

16. Owczarzy R, Vallone PM, Goldstein RF, Benight AS: Studies of DNA dumbbells VII: evaluation of the next-nearest-neighbor sequencedependent interactions in duplex DNA. Biopolymers 1999, 52:29-56.

17. Owczarzy R, Vallone PM, Gallo FJ, Paner TM, Lane MJ, Benight AS: Predicting sequence-dependent melting stability of short duplex DNA oligomers. Biopolymers 1997, 44(3):217-239.

18. SantaLucia J: A unified view of polymer, dumbbell, and oligonucleotide DNA nearest-neighbor thermodynamics. Proc Natl Acad Sci USA 1998, 95(4):1460-1465

19. Doktycz MJ, Morris MD, Dormady SJ, Beattie KL, Jacobson KB: Optical melting of 128 octamer DNA duplexes. Effects of base pair location and nearest neighbors on thermal stability. J Biol Chem 1995, 270(15):8439-8445

20. Allawi HT, SantaLucia J: Thermodynamics of internal C.T mismatches in DNA. Nucleic Acids Res 1998, 26(11):2694-2701.

21. Allawi HT, SantaLucia J: Nearest neighbor thermodynamic parameters for internal G.A mismatches in DNA. Biochemistry 1998, 37(8):2170-2179.

22. Leonard GA, Booth ED, Brown T: Structural and thermodynamic studies on the adenine.guanine mismatch in B-DNA. Nucleic Acids Res 1990, 18(19):5617-5623.

23. Li Y, Zon G, Wilson WD: Thermodynamics of DNA duplexes with adjacent G.A mismatches. Biochemistry 1991, 30(30):7566-7572.

24. Gotoh O, Tagashira Y: Stabilities of nearest-neighbor doublets in doublehelical DNA determined by fitting calculated melting profiles to observed profiles. Biopolymers 1981, 20:1033-1042.

25. Vologodskii AV, Amirikyan BR, Lyubchenko YL, Frank-Kamenetskii MD: Allowance for heterogeneous stacking in the DNA helix-coil transition theory. J Biomol Struct Dyn 1984, 2:131-148.

26. Breslauer KJ, Frank R, Blöcker $H$, Marky LA: Predicting DNA duplex stability from the base sequence. Proc Natl Acad Sci USA 1986, 83(11):3746-3750.

27. Delcourt S, Blake R: Stacking energies in DNA. Journal of Biological Chemistry 1991, 266(23):15160-15169.

28. Doktycz MJ, Goldstein RF, Paner TM, Gallo FJ, Benight AS: Studies of DNA dumbbells. I. Melting curves of 17 DNA dumbbells with different duplex stem sequences linked by T4 endloops: evaluation of the nearestneighbor stacking interactions in DNA. Biopolymers 1992, 32(7):849-864.

29. SantaLucia J, Allawi HT, Seneviratne PA: Improved nearest-neighbor parameters for predicting DNA duplex stability. Biochemistry 1996, 35(11):3555-3562.

30. Sugimoto N, Nakano S, Yoneyama M, Honda K: Improved thermodynamic parameters and helix initiation factor to predict stability of DNA duplexes. Nucleic Acids Res 1996, 24(22):4501-4505.

31. Allawi HT, SantaLucia J: Thermodynamics and NMR of internal G.T mismatches in DNA. Biochemistry 1997, 36(34):10581-10594.

32. Aboul-ela F, Koh D, Jr IT, Martin FH: Base-base mismatches. Thermodynamics of double helix formation for dCA3XA3G + dCT3YT3G (X, Y = A, C, G, T). Nucleic Acids Research 1985, 13(13):4811-4824.

33. Tibanyenda N, De Bruin SH, Haasnoot CA, Marel van der GA, van Boom JH, Hilbers CW: The effect of single base-pair mismatches on the duplex stability of d(T-A-T-T-A-A-T-A-T-C-A-A-G-T-T-G). d(C-A-A-C-T-T-G-A-T-A-TT-A-A-T-A). Eur J Biochem 1984, 139:19-27.

34. Tanaka F, Kameda A, Yamamoto M, Ohuchi A: Thermodynamic parameters based on a nearest-neighbor model for DNA sequences with a singlebulge loop. Biochemistry 2004, 43(22):7143-7150.
35. Wilson WD, Dotrong MH, Zuo ET, Zon G: Unusual duplex formation in purine rich oligodeoxyribonucleotides. Nucleic Acids Res 1988 16(11):5137-5151.

36. Petruska J, Arnheim N, Goodman MF: Stability of intrastrand hairpin structures formed by the CAG/CTG class of DNA triplet repeats associated with neurological diseases. Nucleic Acids Res 1996, 24(11):1992-1998.

37. Allawi HT, SantaLucia J: Nearest-neighbor thermodynamics of internal A.C mismatches in DNA: sequence dependence and pH effects. Biochemistry 1998, 37(26):9435-9444

38. Sugimoto N, Tanaka A, Shintani Y, Sasaki M: Double-Helix Melting of Octamers of Deoxyriboadenylic and Deoxyribothymidylic Acids in the Presence of Ethidium. Chemistry Letters 1991, 20:9-12.

39. A LE, Freier SM: Relative thermodynamic stability of DNA, RNA, and DNA RNA hybrid duplexes: relationship with base composition and structure. Biochemistry 1995, 34(34):10807-10815.

40. Nakano S, Fujimoto M, Hara H, Sugimoto N: Nucleic acid duplex stability: influence of base composition on cation effects. Nucleic Acids Res 1999, 27(14):2957-2965.

41. Seela F, Debelak H: The N(8)-(2'-deoxyribofuranoside) of 8-aza-7deazaadenine: a universal nucleoside forming specific hydrogen bonds with the four canonical DNA constituents. Nucleic Acids Res 2000, 28(17):3224-3232.

42. Panjkovich A, Melo F: Comparison of different melting temperature calculation methods for short DNA sequences. Bioinformatics 2005, 21(6):711-722.

43. Bommarito S, Peyret N, SantaLucia J: Thermodynamic parameters for DNA sequences with dangling ends. Nucleic Acids Res 2000, 28(9):1929-1934.

44. Clark CL, Cecil PK, Singh D, Gray DM: CD, absorption and thermodynamic analysis of repeating dinucleotide DNA, RNA and hybrid duplexes $[\mathrm{d} / \mathrm{r}$ $(A C)] 12 .[d / r(G T / U)] 12$ and the influence of phosphorothioate substitution. Nucleic Acids Res 1997, 25(20):4098-4105.

45. Gelfand CA, Plum GE, Grollman AP, Johnson F, Breslauer KJ: Thermodynamic consequences of an abasic lesion in duplex DNA are strongly dependent on base sequence. Biochemistry 1998, 37(20):7321-7327.

46. LeBlanc D, Morden K: Thermodynamic characterization of deoxyribooligonucleotide duplexes containing bulges. Biochemistry 1991, 30(16):4042-4047.

47. Petruska J, Goodman MF, Boosalis MS, Sowers LC, Cheong C, Tinoco I: Comparison between DNA melting thermodynamics and DNA polymerase fidelity. Proc Natl Acad Sci USA 1988, 85(17):6252-6256.

48. Peyret N, Seneviratne PA, Allawi HT, SantaLucia J: Nearest-neighbor thermodynamics and NMR of DNA sequences with internal A.A, C.C, G. G, and T.T mismatches. Biochemistry 1999, 38(12):3468-3477.

49. Pirrung MC, Zhao $X$, Harris SV: A universal, photocleavable DNA base: nitropiperonyl 2'-deoxyriboside. J Org Chem 2001, 66(6):2067-2071.

50. Plum GE, Grollman AP, Johnson F, Breslauer KJ: Influence of an exocyclic guanine adduct on the thermal stability, conformation, and melting thermodynamics of a DNA duplex. Biochemistry 1992, 31(48):12096-12102.

51. Ratmeyer L, Vinayak R, Zhong YY, Zon G, Wilson WD: Sequence specific thermodynamic and structural properties for DNA.RNA duplexes. Biochemistry 1994, 33(17):5298-5304.

52. Sugimoto $\mathrm{N}$, Honda Kl, Sasaki M: Application of the thermodynamic parameters of DNA stability prediction to double-helix formation of deoxyribooligonucleotides. Nucleosides Nucleotides 1994, 13(6):1311-1317.

53. Wu P, Nakano S, Sugimoto N: Temperature dependence of thermodynamic properties for DNA/DNA and RNA/DNA duplex formation. Eur J Biochem 2002, 269(12):2821-2830.

doi:10.1186/1471-2105-11-105

Cite this article as: Tulpan et al:: Free energy estimation of short DNA duplex hybridizations. BMC Bioinformatics 2010 11:105. 